\title{
L'AMÉNAGEMENT DU TERRITOIRE DE DEUX COMMUNES VAUDOISES: LUTRY ET SAVIGNY, SON ÉVOLUTION
}

\author{
VICTOR RUFFY
}

\section{INTRODUCTION}

Lorsqu'on parle aujourd'hui d'aménagement du territoire on croit souvent à une notion nouvelle exprimant une prise de conscience récente. Rien n'est plus erroné; tout territoire habité a été aménagé selon une optique précise répondant à des contingences de tous ordres mais avant tout de contingences économiques et pouvant revêtir des formes très diverses suivant la combinaison des facteurs en présence. Le plan d'un aménagement peut rester mystérieux lorsque nous manquons de données; il peut également nous apparaître plus ou moins adapté aux exigences du moment, il n'empêche que chaque fois des impératifs se sont trouvés à la base d'un établissement humain. Si en ce moment l'homme est capable de porter un jugement sur la terre tout en s'affranchissant de son attraction, il ne faut pas oublier que nos lointains ancêtres avaient des préoccupations beaucoup plus terre-à-terre, ne pouvant prolonger leur regard que rarement en montant sur un arbre ou en gravissant une colline.

Si de nos jours, l'aménagement du territoire peut faire l'objet d'un ministère, c'est que nos pays subissent une crise due, d'une part à l'éclatement des barrières économiques, d'autre part à un essor industriel fulgurant; les conséquences directes de ces deux phénomènes sont une urgente harmonisation de l'industrie et de l'agriculture avec pour corollaire le problème de l'habitat (développement des villes, urbanisation des campagnes, planification agricole).

Il serait également faux de croire que les crises actuelles sont les premières en date. Elles atteignent certes des proportions jusqu'ici inconnues, mais lorsqu'on étudie ce problème au point de vue historique sur un plan restreint, on s'aperçoit qu'à certaines époques anciennes déjà, certaines sociétés ont fait face à des situations difficiles en mettant en œuvre des mesures touchant l'ensemble d'une région.

Les facilités de transport par la route, le chemin de fer, l'air, la mer permettent de telles migrations contrôlables et incontrôlables, de tels échanges de marchandises et à si longue distance que les régions se sont vues classées très rapidement en régions à économie ouverte et fermée. Il ne s'agit pas de savoir si celles-là ont perdu, en caractère ce qu'elles ont gagné en superficie, contentons-nous de remarquer que les disparités entre ces deux catégories se développent selon une progression géométrique et qu'elles ont suscité de la part des hommes une recherche qui a abouti à la «regional science».

On pourra alors s'interroger sur la valeur d'un travail portant sur de si petites régions et qui s'ouvre très modestement sur la période moderne. Il nous a paru cependant utile de tenter de dégager quels ont été les facteurs principaux qui ont présidé à l'élaboration de l'aménagement d'un territoire certes petit mais qui représente pourtant deux évolutions totalement différentes dont l'une est directement dépendante de l'autre. En effet, autrefois complémentaire ces deux régions sont pratiquement sans liens de nos jours. C'est ce phénomène que nous essayerons de saisir.

\section{LE RELIEF ET LE CLIMAT: \\ FACTEURS PRIMORDIAUX DANS L'AMÉNAGEMENT DU TERRITOIRE}

S'étendant à l'extrêmité sud-est du plateau vaudois et sur sa retombée dans le bassin lémanique, la région étudiée repose entièrement sur des couches molassiques plongeant vers les Alpes; les têtes de ces couches sont orientées Sud-Ouest - Nord-Est. Notons en passant l'opposition entre la direction des têtes de couches et le pendage qui est lui Nord-Ouest-Sud-Est, elle nous aidera plus tard à comprendre l'hydrographie de la région. 


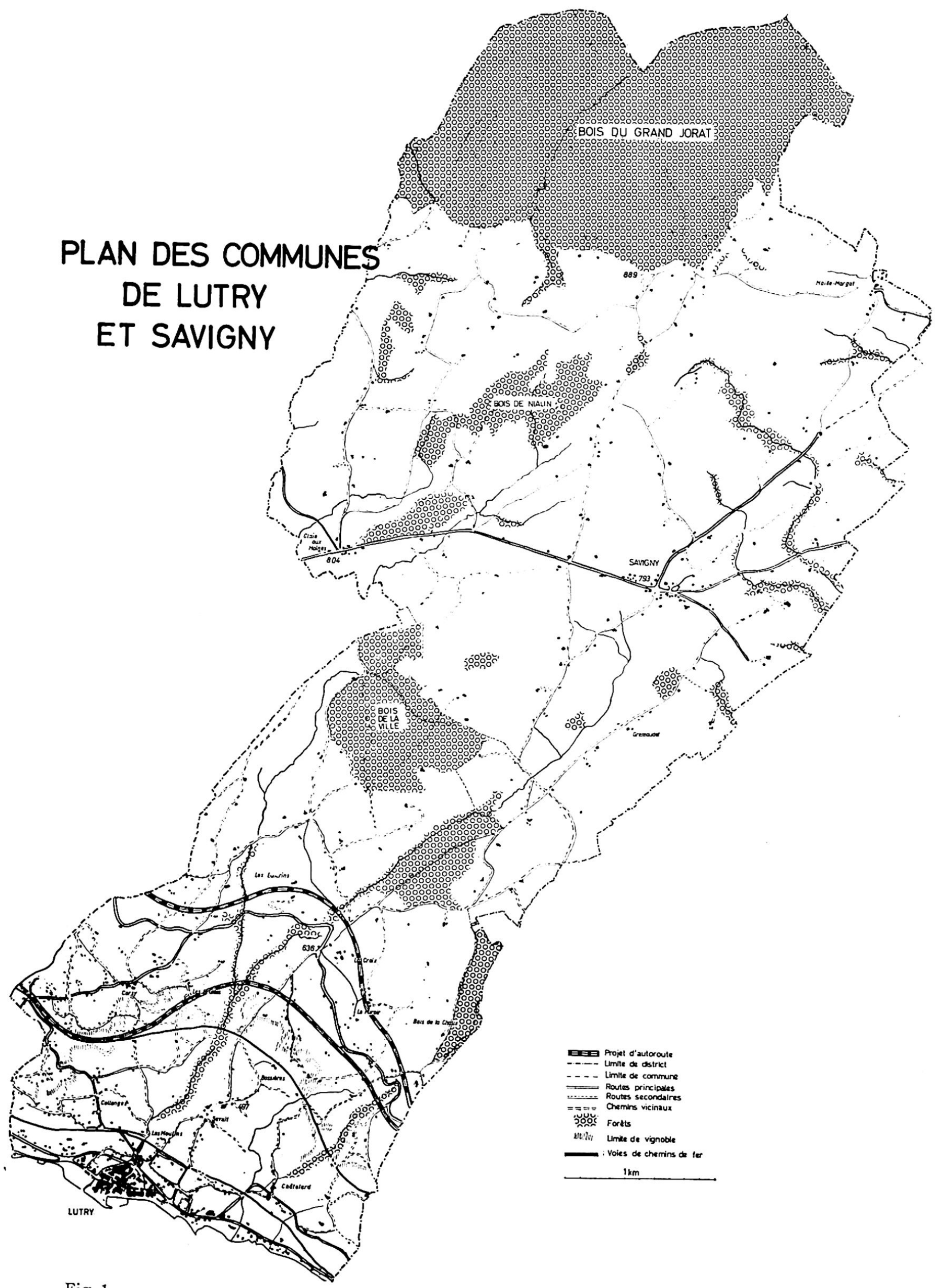

Fig. 1 


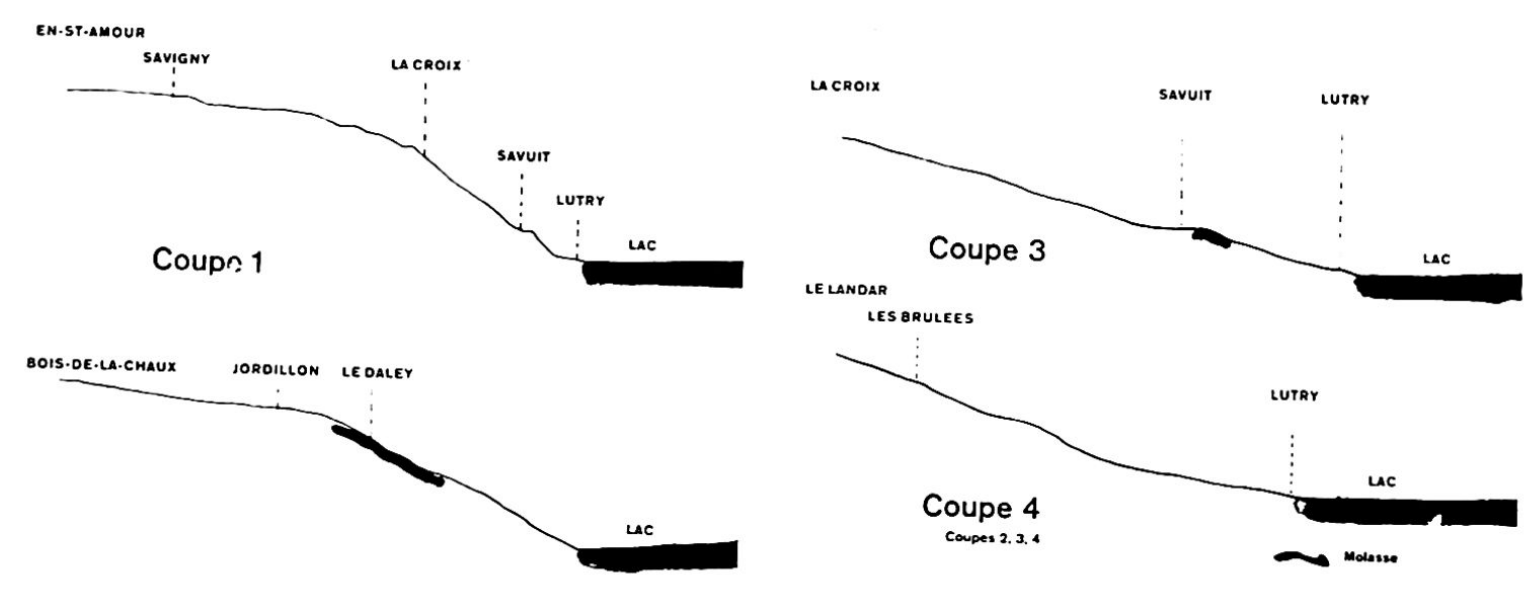

Figure 2

M. A. Bersier ${ }^{1}$ a très bien démontré la nature structurale du plateau en prouvant que les prétendus drumlins n'étaient que des cuestas émoussées, et que les accidents de Savuit, Bochat, Aran ne sont que de têtes de bancs. Elles sont parentes des cuestas du plateau quoique plus travaillées à cause de la pente beaucoup plus raide. En effet, si l'on fait une coupe à travers notre région, on s'aperçoit que malgré la parenté du sous-sol, nous avons deux régions distinctes l'une de l'autre par leur modelé. Pour l'une l'action du glacier a été capitale, elle a créé sa pente, déterminé sans hésitation son orientation vers le Sud, constitué en grande partie ses sols superficiels, pour l'autre elle fut moins active, déposant par-ci, par-là, des matériaux morainiques (alluvions, argile). L'une des régions appartient à l'auge proprement dite du glacier, l'autre à la région marginale. A partir du niveau du lac $(372 \mathrm{~m}$.), la pente est à peu près de $13 \%$ jusqu'à $2 \mathrm{~km}$. de la rive, puis à partir de la cote $630 \mathrm{~m}$. il faut à peu près $5,5 \mathrm{~km}$. pour atteindre les cotes $880 \mathrm{~m}$. Ces chiffres montrent bien la différence fondamentale de déclivité entre les deux régions (voir fig. 2).

$\mathrm{La}$ région nord appartient certes au plateau, mais il faut cependant noter des altitudes élevées qui gravitent autour de $800 \mathrm{~m}$. avec comme point culminant les Côtes $906 \mathrm{~m}$. (N. de Savigny), ce qui est très haut pour notre plateau. De plus ce terme de plateau ne doit pas nous faire penser dans le cas présent à une surface plane; incliné, parcouru par des fronts de côtes émoussés, ce territoire présente un aspect passablement accidenté et compartimenté par une hydrographie tantôt subséquente, tantôt conséquente. Ceci est valable surtout dans la région ouest; à l'est en effet le relief semble plus plat, ouvert en direction de Forel. Il importera de se souvenir de cette structure lorsqu'on abordera le chapitre des voies de communication.

La région sud n'a pas une pente uniforme et sur la commune de Lutry on peut déjà constater d'importantes différences; affleurements, bombements de molasses l'accentuent alors que dans d'autres secteurs (Nord de Savuit, Bossières) une épaisse couche de dépôt morainique l'atténue. Cette différence de pente, cette différence de nature du sol sont autant d'éléments de différenciation dans une région viticole. Quant à l'hydrographie, elle est sensiblement plus simple que dans la région nord, ici plus d'hésitation, la direction est bien marquée, d'autant plus qu'à cause de la pente les sols superficiels ont rapidement été entraînés et que les cours d'eau ont pu très tôt attaquer la couche molassique et former des gorges souvent profondes.

Avec une telle dénivellation entre le niveau du lac $(372 \mathrm{~m}$.) et le point culminant de la commune de Savigny $(906 \mathrm{~m}$.) on doit forcément s'attendre à des climats très dif-

1 Bulletin des laboratoires de géologie, minéralogie, géophysique et du musée géologique de l'Université de Lausanne, Bulletin no. 75, Lausanne 1942. 
férents. Nous ne possédons malheureusement pas de données propres aux deux communes, mais grâce aux travaux de Monsieur P.-L. Mercanton² nous pourrons extrapoler.

Pour notre région, deux éléments fondamentaux sont à retenir en ce qui concerne le climat: différence d'altitude et influence de la nappe lémanique.

La température moyenne annuelle pour la région de Lausanne s'élève d'après $\mathrm{H}$. Dufour ${ }^{3}$ de $0,58^{\circ}$ pour 100 m., ce qui fait pour Savigny $7,56^{\circ}$. Si l'on établit les températures moyennes annuelles pour les autres régions on trouve pour La Croix $636 \mathrm{~m}$. $8,47^{\circ}$, pour Savuit $467 \mathrm{~m} .9,45^{\circ}$, pour Lutry $9,99^{\circ}$. Il y a donc plus de deux degrés de différences entre les deux moyennes annuelles de Lutry et de Savigny. Il serait bon de pouvoir comparer les amplitudes mensuelles. Là encore les données nous manquent, mais l'on peut avancer sans trop de risque que les amplitudes sont beaucoup plus fortes à Savigny, que ce soit durant vingt-quatre heures, une saison ou encore une année. Les hivers surtout sont plus marqués, le printemps et l'automne passent très vite. Razoumovsky ${ }^{4}$ notait déjà des changements de temps brusques, la fréquence des orages en été et les grands froids en hiver. Il relevait encore une grande pluviosité en automne et au printemps. Alors qu'au bord du lac la température du sol est très fortement influencée par celle de ce dernier, (jusqu'à la courbe 400 la neige ne reste pas et il est rare qu'elle tombe à Lutry même) à Savigny l'épaisseur du manteau peut atteindre jusqu'à $1,50 \mathrm{~m}$. et recouvrir la contrée durant trois mois. La date des premières neiges se situe vers la fin octobre et celle des dernières vers la fin avril, début mai, même. Si l'une des communes endure un long hiver, elle n'a pas, en contrepartie, à souffrir des gelées de printemps comme l'autre. Bien que depuis 1938 il n'y ait pas eu de gelées de printemps à proprement parler dans le vignoble, il suffit de remonter à 1933 pour trouver les méfaits catastrophiques de ces dernières. Le cas de 1956 doit être mis à part, ce ne fut pas des gelées de printemps (c'était au mois de février) mais malheureusement le temps ayant été doux auparavant, le gel endommagea les ceps où la sève était déjà montée. Il n'y a pas seulement le gel qui est craint ici, et sans conséquence là-bas, il y a encore la grêle presque inconnue à Savigny, elle est la sorcière du vignoble, venant presque toujours avec le Joran, de Belmont ou alors du lac selon un axe Nord-Ouest - Sud-Est; elle anéantit avec caprice certaines régions en en épargnant d'autres. Depuis longtemps l'homme cherche à la déjouer, on tirait autrefois du canon, depuis trois ans les vignerons lâchent des fusées. On sait quels ont été les dégâts de l'année dernière. Bien que l'utilité des fusées ne soit plus mise en doute, il faudra encore quelque temps jusqu'à ce qu'on les lâche au bon moment et à l'endroit judicieux.

Le régime des pluies est un facteur déterminant pour la végétation et la conservation des sols. Monsieur P. L. Mercanton ${ }^{5}$ a fait une étude comparée des chutes de pluie depuis le niveau du lac jusqu'en Gourze. Il fit la même expérience plus tard à Lausanne. Dans les deux cas, les résultats prouvèrent une nette augmentation de la pluviosité à mesure qu'il s'éloignait du lac. A l'aide des deux barèmes nous avons calculé une répartition approchée de la pluie aux diverses altitudes

$\begin{array}{lll}380 \mathrm{~m} & \text { Lutry } & 1023 \mathrm{~mm} \\ 460 \mathrm{~m} & \text { Savuit } & 1084 \mathrm{~mm} \\ 636 \mathrm{~m} & \text { La Croix } & 1180 \mathrm{~mm} \\ 802 \mathrm{~m} & \text { Savigny } & 1257 \mathrm{~mm}\end{array}$

2 Bulletins de la Société Vaudoise de Sciences Naturelles, no. 282 (Climatologie lausannoise) et no. 233 (Le régime pluvial de Cully à la Tour de Gourze). Lausanne, 1952.

3 Ch. Biermann, Le Jorat. Neuchâtel 1910, page 42.

4 G. de Razoumovsky, Histoire naturelle du Jorat et de ses environs, II volumes, Lausanne, 1789 , tome II, page 6.

5 P.-L. Mercanton. Le régime pluvial de Cully à la Tour de Gourze. Bulletin de la Société vaudoise des sciences naturelles. Lausanne. Imprimerie Comm., 1933. 


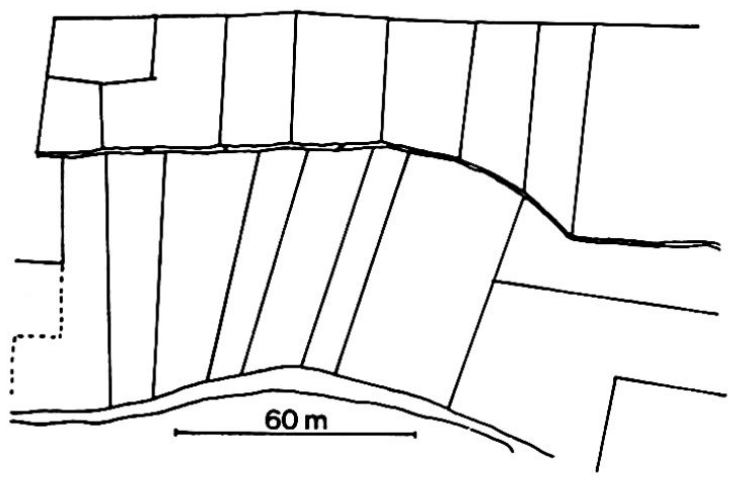

Figure 3

Parcellement régulier du vignoble

Plus que les pluviosités annuelles, ce sont les graphiques pluviométriques qui sont intéressants ${ }^{6}$. En effet, on constate que les maxima à Lausanne comme à Cully se situent entre août et septembre, alors que les minima sont en général entre janvier et mars. Il est assez significatif que lors de nos enquêtes chez les paysans et les vignerons, ceux-ci n'ont presque jamais mentionné août et septembre comme étant les mois les plus pluvieux. Cette constatation nous amène à considérer la nature des chutes de pluie estivales. Il s'agit en effet de pluies d'orage, extrêmement fortes et subites. L'orage vient souvent de la Dent d'Oche ou encore de la Dranse, vers les cinq, six heures du soir et dure alors jusqu'à sept, huit heures. Ces pluies, si elles sont souvent bénéfiques pour les raisins, mettent à mal les coteaux, emportant de la terre par ruissellement, fissurant des murs, provoquant des glissements de terrain (mouvements des dépôts glaciaires sur le soubassement molassique). On voit alors les ruisseaux rouler des eaux boueuses et on constate l'indispensable rôle que jouent les drains à ciel ouvert, qu'on nomme «les coulisses». Si la neige a été et reste encore le principal perturbateur de la circulation à Savigny c'est l'eau qui tient ce rôle dans la commune de Lutry.

En ce qui concerne le régime des vents, nous n'avons pas trouvé de documents scientifiques écrits et nous avons dû nous référer aux données des vignerons et des paysans interrogés et aux renseignements fournis par Ch. Biermann ${ }^{7}$. A Savigny l'action de la bise peut considérablement perturber la circulation; c'est elle, en effet, qui règne durant l'hiver, soufflant la neige dans les combes et sur les routes. Le système de palissades à claire-voie orientées perpendiculairement à la direction des vents, mentionné par Ch. Biermann ${ }^{8}$ est toujours employé. Si l'on observe la carte nationale au 1:25000, on s'aperçoit qu'aux alentours de Savigny une triple rangée d'arbres borde des ruisseaux sur leur cours conforme au pendage, c'est-à-dire perpendiculaire à l'axe des vents dominants. Il semble bien que le maintien de ces bois peu rentables soit une mesure de protection. Le vent d'été par excellence est le vent de Genève apportant généralement la pluie. D'importance secondaire sont le vent blanc, la bise noire, le Joran et la Vaudaire.

Les deux vents dominants sont les mêmes à Lutry et à Savigny. La bise souffle pratiquement à toutes les saisons; elle est surtout redoutée au printemps où elle peut arracher les bois tendres d'une végétation tardive. Quant au vent, c'est lui qui souffle au printemps et accompagne les orages d'été. On comprend mieux maintenant les graphiques pluviométriques. Les vents moins fréquents mais dont les conséquences peuvent être très grandes sont le Joran, porteur de grêle parfois et la Vaudaire tiède qui vient de la vallée du Rhône et qui est toujours bien accueillie.

6 P.-L. Mercanton. Climatologie lausannoise. Bulletin de la Société vaudoise des sciences naturelles. Lausanne, Imprimerie Baud, 1952.

7 Ch. Biermann. Le Jorat. Neuchâtel, Attinger, 1910. Page 41.

8 Ch. Biermann. Le Jorat. Neuchâtel, Attinger, 1910, page 46.

9 Carte nationale au 1:25 000, feuille no. 1243 . 
«Vaudaire du matin fait tourner les moulins.

Vaudaire du soir fait tourner les pressoirs.»

Le régime des brouillards est différent pour les deux communes, la nature marécageuse du terrain autour de Savigny, les Mollies, la Goille favorise la formation de nappes localisées de brouillard même pendant les nuits d'été. Durant tout l'automne, il reste le principal ennemi pour les automobilistes. En revanche, il est rare que le vignoble soit dans le brouillard, le niveau le plus bas de la couche se situant d'habitude à la cote $550 \mathrm{~m}$.

\section{L'ÉLÉMENT HUMAIN}

Les bords du Léman furent habités depuis la préhistoire. A plusieurs reprises on a trouvé au Châtelard sur la commune de Lutry des tombes néolithiques. Nous avons ainsi la certitude que Lutry et ses environs furent peuplés très tôt. Les Ligures, les Celtes, les Helvètes, les Romains au 1er siècle avant J.-C. et les Burgondes à partir du Ve siècle succédèrent aux premiers occupants ${ }^{10}$. On sait peu de choses sur le paysage de cette époque; il est logique de penser que la vigne poussait au temps des Romains, mais les documents pour nous le prouver sont minimes et leur rareté nous indique que son extension était réduite. Le premier acte faisant mention de la vigne date de 906 , on y trouve un cens en nature, composé entre autres d'un setier de vin. En 997 une donation entre vifs est faite par le roi Rodolphe à son chancelier d'une vigne à Lutry et de plusieurs autres terres. Ce sont ces terres de Lutry qui seront données en 1025 au monastère de Savigny situé à $20 \mathrm{~km}$. au Nord-Ouest de Lyon. C'est à partir de cette époque également que la puissance de l'Evêque de Lausanne ne cesse d'augmenter. Droit de juridiction donné par l'Empereur, transactions avec le prieuré de Lutry, don de l'abbaye de St-Maurice et achat de propriétés font de l'Evêque le plus puissant et le plus grand propriétaire de la commune de Lutry.

\section{Le clergé et la politique du vin}

Dans l'introduction de son ouvrage, R. Dion ${ }^{11}$ analyse les facteurs favorables à l'aménagement d'un vignoble au moyen-âge. Pour les vignobles restreints la présence d'établissements religieux suffisait. Les couvents possédaient ainsi leur propre vin de messe, même s'il n'était pas d'une bonne qualité. Pour les vignobles de grandes dimensions la situation par rapport à l'environnement entre en jeu. L'auteur voit trois situations favorables:

1. près de grandes villes et des rivières navigables qui les desservent;

2. près de la mer

3. près des montagnes froides.

Si l'on examine la situation du Lavaux en fonction de ces critères, on s'aperçoit que cette région est particulièrement privilégiée. La présence d'une ville épiscopale à moins de $20 \mathrm{~km}$., ville épiscopale petite, certes, mais tête d'étape cependant sur la grande artère France-Italie, offrait des débouchés à une production viticole. L'existence d'une bourgeoisie artisanale, la tradition des marchés bi-hebdomadaires assuraient une certaine consommation. La lecture du Plaict général de Lausanne ${ }^{\mathbf{1 2}}$ nous en fournit la preuve par les articles qu'il contient sur le commerce du vin. Quant à la situation par rapport

10 A.-M. Courtieu. La vigne dans la partie méridionale de l'ancien diocèse de Lausanne au moyen âge. Paris, Ecole des Chartes, 1959, page 2.

11 R. Dion. Histoire de la vigne et du vin en France des origines au XIXe siècle. Paris, 1959. Introduction, page 35 et ss.

12 J.-P. Baud. Le Plaict général de Lausanne. Lausanne 194o, page 273, articles 107-113. 


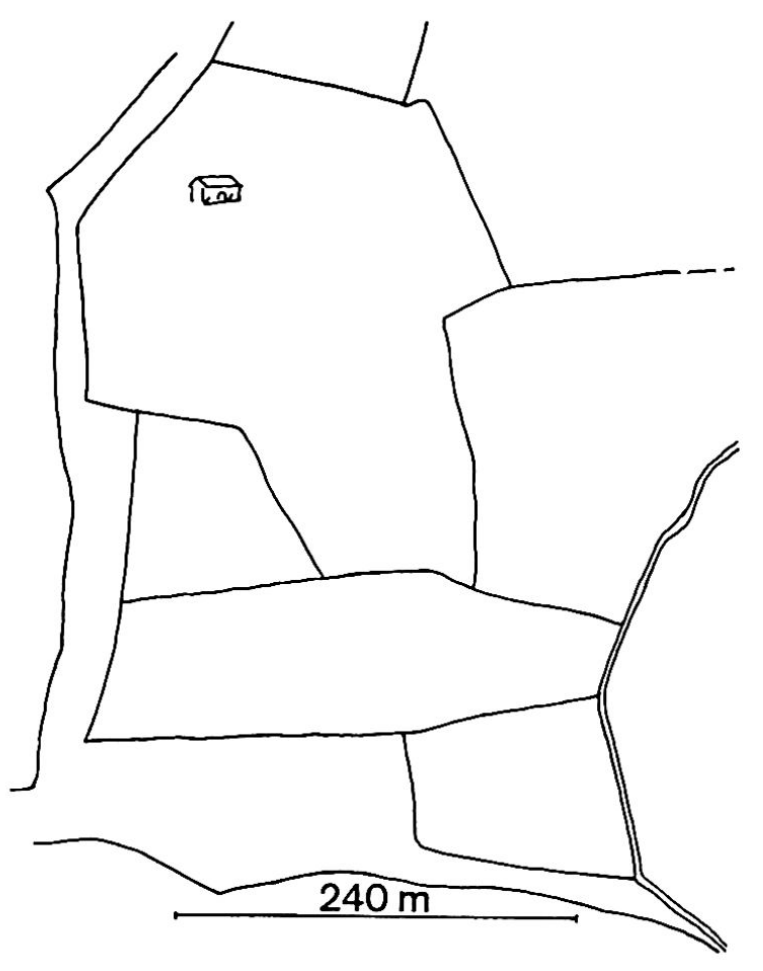

Figure 4

Parcellement anarchique des * Hauts»

aux montagnes froides, Lavaux est particulièrement privilégié, le lac permettant à ses producteurs de faire parvenir leur vin au pied de celles-ci sans gros frais. Les principaux débouchés se trouvaient cependant sur le plateau vaudois, dans la vallée de la Broye. Moudon, Payerne, Cugy près Payerne, Combremont-le-Grand, Bioley-Magnoux, Fribourg et la Gruyère sont ${ }^{13}$ les régions mentionnées dans des actes de vente. Toutes ces conditions favorables ne pouvaient que stimuler l'acharnement au travail des Bénédectins établis à Lavaux et dotés de terres à condition de les défricher et de les transformer en vignoble «ad vineam edificandam ed faciendas vineas». Il est très difficile comme nous l'avons déjà dit de donner une description précise du pays avant l'arrivée des moines et de mesurer l'exacte transformation. Une analyse scientifique du sol encore à faire permettrait sans doute de déterminer le genre de végétation qui a précédé la vigne. On peut cependant pour l'instant penser sans trop de risque d'erreur que les coteaux devaient être recouverts d'herbe, de broussailles et de quelques terres cultivées d'une manière extensive. Pour pratiquer une culture intensive dans une région comme la nôtre et avec ses conditions climatiques, il fallait un aménagement préalable du territoire.

\section{L’aménagement du vignoble}

Le vignoble de Lavaux sous sa forme actuelle est une région aménagée résultant d'une culture intensive. Madame Courtieu ${ }^{14}$ a recherché avec soin dans les minutes de notaire la première apparition des termes propres à notre vignoble actuel.

En ce qui concerne l'aménagement des terrasses l'auteur a trouvé en 1331 le mot «charmu» qui désigne le mur qui soutient la terre en pente et dont l'étymologie serait «calvus murus». La signification actuelle de surface étroite de terre inclinée n'est qu'une extension du terme ancien. A la fin du XIVe siècle on retrouve ce mot dans un acte

13 A.-M. Courtieu-Capt. Recherches sur l'histoire du vignoble au moyen âge dans la partie méridionale de l'ancien diocèse de Lausanne. Paris, Ecole des Chartes, 1959. (Epreuve polycopiée des Archives cantonales vaudoises). Pages 142-145.

14 A.-M. Courtieu-Capt. Recherches sur l'histoire du vignoble au moyen âge dans la partie méridionale de l'ancien diocèse de Lausanne. Paris. Ecole des Chartes, 1959. Page 15 et 16. 
passé entre des citoyens de Lutry. La nécessité de ces constructions était indiscutable puisqu'on n'omettait pas dans les contrats de mentionner la réfection de ces murs, comme obligation pour le vigneron.

On rencontre le mot «coulisse» qui désigne le canal perpendiculaire au lac chargé de collecter les eaux de ruissellement; en 1392, il est écrit dans un contrat

«debet quondam collesiam, debet ( $\mathrm{sic}$ ) et tenetur nemus dicte collesie extirpere et trahere et dictae collesiam mondare».

Le fait d'insister sur la propreté des coulisses montre le rôle indispensable qu'elles jouaient déjà à cette époque. L'auteur de la thèse relève encore la formule intéressante d'un contrat «item refacere collisias dictarum vinearum si depresenti alique sint refaciende et semper cum replete fuerunt seu destructe». Il faut donc refaire les coulisses lorsqu'elles sont bouchées; il semble que ce ne fût pas de véritables constructions, mais de simples fossés aidant également à délimiter les parcelles. L'action de l'eau de ruissellement sur les sols démunis d'arbres et de buissons après la plantation de la vigne dut causer rapidement la descente des terres. Aussi dès la fin du XIVe siècle on peut lire dans les contrats l'expression «portare terram» qui implique l'obligation pour l'exploitant de reporter dans la partie supérieure de la vigne la terre accumulée pendant deux ou trois ans dans sa partie basse. On maintenait ainsi, comme on maintient aujourd'hui encore la surface cultivable inclinée, tout en soulageant le mur inférieur.

Quant aux soins portés à la vigne, on s'aperçoit qu'ils étaient presque aussi nombreux qu'aujourd'hui, la lutte contre les maladies mise à part. On lit dans les minutes de notaires le verbe «putare» de «amputare» qui veut dire tailler, puis «propagare», provigner; aucun contrat ne nous donne de détails sur la technique de la reproduction des plants, il faut cependant penser à la signification actuelle du terme pour en déduire qu'on employait le marcottage, procédé le plus naturel en vogue dans tous les vignobles d'alors. On fixe dans les contrats la fréquence des provignages, ils doivent en général avoir lieu tous les cinq, six ans, ce qui montre que les plants étaient moins vigoureux qu'aujourd'hui; la durée d'un plant de vigne étant de nos jours de quinze ans. On rencontre ensuite «fodere» ou «fosserare», qui a donné notre fossoyer et notre unité de surface le fossorier, espace de terrain qu'un homme peut en une journée travailler au fossoir $\left(450 \mathrm{~m}^{2}\right)$. Le travail ensuite mentionné est «facere folia» trois fois l'an, ces trois opérations coïncident avec nos «éplanage», «rebiolage», «biochage».

Un point important est de savoir comment poussait la vigne. Nous n'avons pour notre part encore jamais trouvé le mot hutain sur la commune de Lutry. Bien qu'on ait la certitude que des arbres poussaient dans les vignes (il existe des contrats qui partagent en deux les fruits de la vigne et aussi ceux des arbres qui s'y trouvent ${ }^{15}$ ) ils n'étaient pas en quantité suffisante pour soutenir les sarments. Il faut penser alors que la vigne devait soit déjà avoir recours aux échalas, soit pousser sur le sol. A la fin du XIVe siècle, un homme de Bossières doit livrer à un bourgeois de Lutry, avant la prochaine fête de la Purification de la Vierge, quinze douzaines de «fassellorum lianz et marchian en passions vinearum dicti Mermeti». Nous croyons cependant que les vignes étaient loin de présenter l'alignement impeccable d'échalas comme de nos jours. Le simple procédé du marcottage devait entraîner une certaine anarchie, de plus il faut supposer qu'on attachait au même échalas les sarments de plusieurs souches et qu'on plantait beaucoup moins serré. Un contrat très tardif de $1833^{16}$, passé entre la commune de Savigny et son vigneron, stipule à l'article 7: «Le vigneron fournira et maintiendra tous les échalas nécessaires aux vignes, à l'entière décharge de la commune et au contentement des Inspec-

15 A.-M. Courthieu-Capt. Recherches sur l'histoire du vignoble au moyen âge dans la partie méridionale de l'ancien diocèse de Lausanne. Paris, Ecole des Chartes, 1959, page 39.

16 Tiré du livre des conditions de la commune de Savigny de 1833. 
teurs ou commis de la Municipalité savoir: un millier par pose chaque année qu'il fera voir aux Préposés».

Si l'on calcule la pose à $4500 \mathrm{~m}^{2}$ selon les données de G.-A. Chevallaz ${ }^{17}$, on s'aperçoit que le nombre des échalas au début du XIXe siècle était quatre fois et demi inférieur à celui de nos jours; cette constatation laisse à penser qu'il devait être inférieur encore durant les premiers siècles du vignoble. L'importance très minime du problème de l'attachage semble prouver nos dires. Bien qu'on relève dans les comptes concernant les vignes des moines du Haut-Crêt «pro aducere fenum et pall» où pall viendrait de palea, cette expression est l'unique mention de cette époque, et il faut peut-être en déduire que les plants d'alors plus vigoureux s'entortillaient d'eux-mêmes aux échalas et que les autres rampaient simplement.

La reconstitution des terres posait déjà les mêmes problèmes de fumure qu'actuellement. A la fin du XIVe siècle un contrat contient l'obligation pour le vigneron de mettre «sex carigatas fimi in curtina sua» dans une vigne pendant six ans 18 .

Ainsi la région présente les caractères d'un paysage transformé progressivement, où une culture intensive prend une extension rapide, si rapide qu'au XIVe siècle la grande cour séculière de Lausanne qui était un conseil communal étendu, interdit de faire entrer ou de vendre en ville n'importe quel vin qui ne venait pas du baillage de Lausanne ou de la région de Lavaux jusqu'a Vevey, à moins qu'il ne s'agisse de vin tiré des vignes possédées par des bourgeois de Lausanne personnellement ${ }^{19}$.

Mais avant de considérer les conséquences économiques extérieures de cette monoculture, il est bon d'abord d'examiner les transformations internes qu'elle a entraînées dans le cadre communal.

\section{LES CONSÉQUENCES INTERNES DE L'EXTENSION DU VIGNOBLE}

\section{Lutry - commune vaste}

Les 2438 hectares de l'ancienne commune de Lutry faisaient d'elle une des villes les plus riches du Pays de Vaud. Il est presque constant dans l'histoire du moyen âge, que les possessions ont développé le sentiment de solidarité des bourgeois contre l'autorité supérieure soit cléricale, soit aristocratique. La possession de pâturages et de vastes forêts sur les Monts obligea les habitants de Lutry à créer très tôt une organisation communale afin d'en faire profiter chaque bourgeois d'une façon équitable et de défendre les intérêts de la communauté vis-à-vis de l'Evêché de Lausanne, du Prieuré de Lutry, ainsi que des communes voisines. Le conflit de 1330 entre les bourgeois de Lutry et la ville de Lausanne au sujet des bois des Râpes 20 en est une preuve.

L'extension du vignoble ne pouvait que renforcer cette solidarité. En effet, d'une part on allait devoir exclure le bétail des endroits réservés à la culture de la vigne et lui trouver de nouveaux pâturages plus haut, ce qui nécessitait un renforcement de la surveillance du troupeau communal, d'autre part on allait devoir exploiter les forêts pour les besoins de l'économie viticole. La construction des pressoirs en bois, celle des tonneaux en chêne, sapin, frêne nécessitèrent un défrichage rapide. Les Monts de Lutry et la région de l'actuelle Savigny s'y prétaient d'ailleurs à merveille, offrant une très grande variété d'essences et ceci sur une vaste superficie qui s'étendait du Nord de la commune actuelle de Savigny jusqu'à la limite actuelle de la vigne entre les cotes 550 et

17 G.-A. Chevallaz. Aspects de l'agriculture vaudoise à la fin de l'ancien Régime. Lausanne, 1949 , page 24 .

18 A.-M. Courtieu-Capt. Recherches sur l'histoire du vignoble au moyen âge dans la partie méridionale de l'ancien diocèse de Lausanne. Paris, Ecole des Chartes, 1959, page 23.

19 M. Reymond. Le vignoble vaudois à travers les siècles, Lausanne 1933, page 20.

20 Dictionnaire historique, géographique et statistique du canton de Vaud, E. Mottaz, Lausanne 1921, tome II, page 173 . 


\section{Plan de la Ville de Lutry 1705}

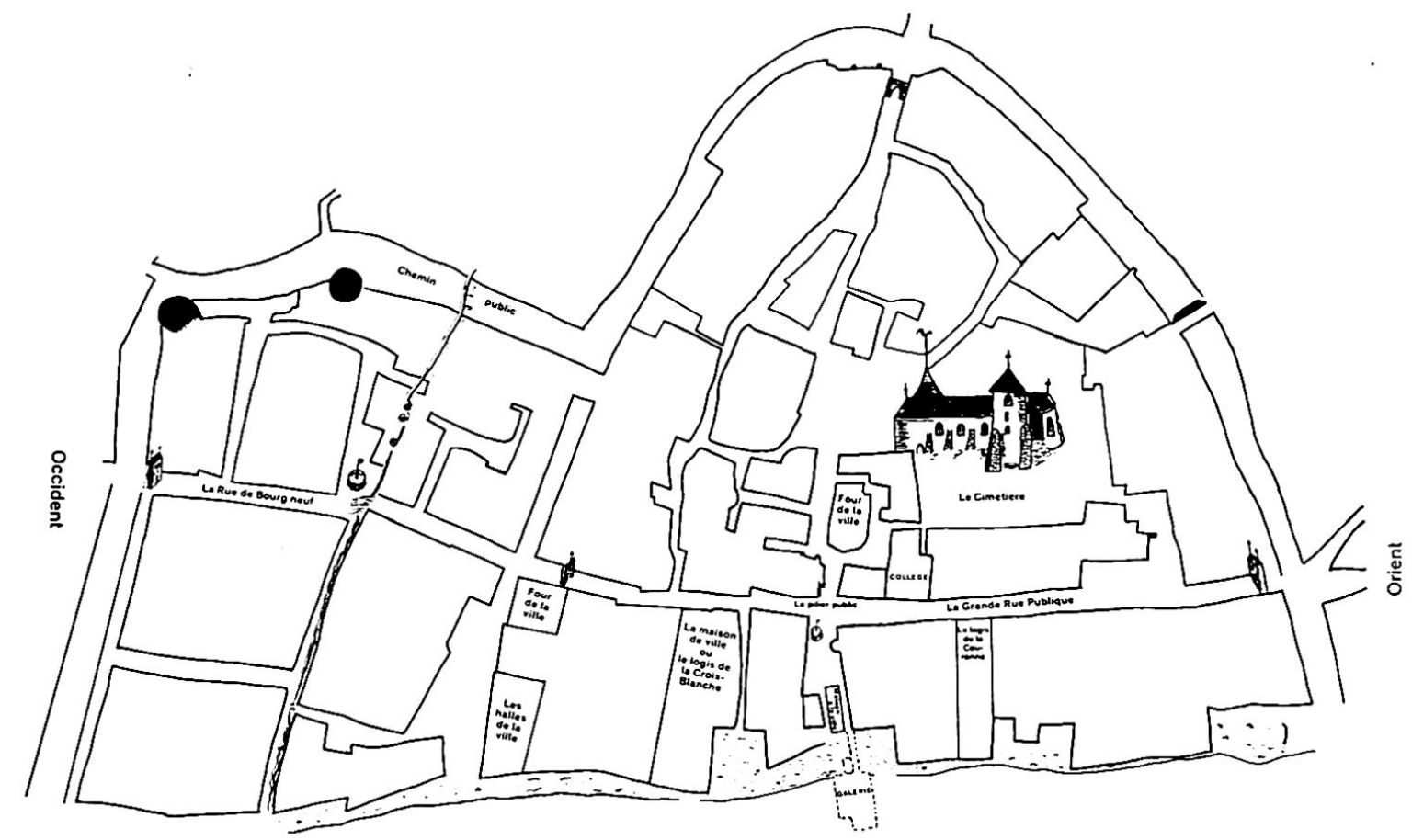

Figure 5 Schéma d'après les plans généraux de la Paroisse de Lutry 1705

600. La toponymie à ce sujet nous est précieuse. On trouve en effet vers cette ancienne limite méridionale des noms de lieux qui attestent l'existence d'anciennes forêts: les Brûlées, Bossières, les Chênes, le Chaney, puis le Daley, qui veut dire bois de dailles (pin sylvestre), puis si l'on va plus à l'intérieur on rencontre les Bioles (lieux où abondent les bouleaux), les Publoz (lieux où croissent les peupliers) et enfin la Saujalle. Razoumovsky relevait la présence de ces forêts de pins sylvestres, de pins épicéa ${ }^{21}$, de hêtres et de chênes le long des lisières méridionales et occidentales aux environs de Rovéréaz et de Savigny. L'on peut dire que le pays est couvert de vignobles presque partout où il n'est point boisé et où l'on a pu placer un plant de vigne.

\section{Lutry - ville forte}

C'est à partir du XIIIe siècle que Lutry devint une ville forte grâce à l'Evêque de Lausanne. C'est un plan de 1705 qui nous permet de retrouver son aspect premier (voir fig. 5). Elle a la forme d'un fer à cheval, ayant deux transversales l'une perpendiculaire et l'autre parallèle au lac. Le pourtour de l'agglomération est souligné par de hauts remparts et des fossés qu'on ne pouvait franchir qu'à quelques endroits. Une porte se trouvait à chaque issue de la ville. La double porte du côté ouest indique que le quartier qui se trouve entre ces deux constructions est d'origine plus récente. Le nom de l'artère principale du dit quartier «Rue du Bourg neuf» confirme cette constatation. Il était particulièrement bien défendu; les fossés normaux étaient parfois doublés de fausses braies.

21 G. de Razoumovsky. Histoire naturelle du Jorat et de ses environs. 2 volumes, Lausanne, Mourer, 1789, tome II, page 8. 
Les remparts sont flanqués d'une tour actuellement encore visible et de la masure d'une ancienne tour. Il est très difficile d'après le plan de savoir si la ville était fortifiée du côté du lac. Il serait vraiment paradoxal qu'on ait fortifié tout le territoire du côté terre et qu'on l'ait laissé ouvert du côté lac; c'était pourtant le cas comme on le verra par la suite.

A côté de son système de défense remarquable, la ville de Lutry nous frappe par son équipement communal; en 1402 elle reçut en abergement de l'évêque, deux fours situés entre la rue principale et le lac, en 1408 l'évêque céda à la ville une place pour y construire une halle et tous les droits de hallage pour le prix de 63 livres lausannoises. Au XVe siècle Lutry possède sa maison de ville. Maxime Reymond dit ${ }^{22}$ : «Dès qu'une ville a été clôturée, les bourgeois ont la charge de l'entretien des remparts, leur intérêt est de s'assurer de l'amenée de l'eau potable, d'entretenir les conduites et les chemins, ils se trouvent en outre dans la nécessité de se défendre contre les voisins et même contre le seigneur qui a des obligations envers eux, ou contre les abus de ses fonctionnaires.»A Lutry deux fontaines sont sur des canaux de dérivation. Un aqueduc dont la construction fut nécessité par le creusement du fossé à un niveau inférieur à celui du ruisseau se trouve au Nord du quartier du Bourg neuf. Ceci nous amène à relever la présence des moulins, il y en a trois à l'intérieur des murs dans ce quartier du Bourg neuf, qui semble avoir une vocation artisanale. Si l'on compare le plan de 1705 à celui de 1810 on s'aperçoit que le cours d'eau qui alimente ces moulins a été en partie recouvert; aujourd'hui on ne remarque plus mềme l'existence de ce ruisseau, et la rue qui se trouve au-dessus de son cours s'appelle la Rue des Tanneurs. C'est donc dans ce quartier que s'est développé l'industrie du cuir jusqu'à la fin du siècle passé. Les moulins de la ville ne sont pas les seuls; sur le même ruisseau on en dénombre encore cinq, un peu plus au Nord dans une région qui s'appelle de nos jours encore «es moulins». L'eau était certes la principale source d'énergie, mais elle n'était pas la seule; nous avons découvert un moulin à vent situé en dehors de la ville au bord du lac ${ }^{23}$, afin de profiter des vents lacustres et de simplifier le problème du transport du grain. Le dicton cité page 5 , trouve donc sa justification.

Ainsi, Lutry par son aspect et son équipement présente les caractères d'une ville où la société est déjà différenciée. Il est certain que les maisons avec étable et courtil prédominent, mais il semble certain aussi que les maisons se trouvent surtout dans le Nord-Est de la ville, les artisans occupent le Bourg neuf alors que la population aisée semble déjà habiter le Sud-Est de la ville, en bordure du lac d'où l'on jouit de la plus belle vue. Il n'est donc pas étonnant que l'auberge se trouve dans ce quartier. Cette hiérarchisation s'explique d'ailleurs aisément; la production du vin et son transport entrainent un artisanat; la construction d'outils, de tonneaux, celle des chars et des bateaux sont indispensables. Les vignerons ont besoin de cordes, de lanières de cuir. L'activité des halles dépend de gens disponibles pour les transbordements, les convois. Cette activité accessoire au début deviendra rapidement un métier. A l'autre extrémité de l'échelle sociale une catégorie de propriétaires s'enrichit et commence à exiger un certain confort et un certain luxe.

Le clivage dans la société est vérifié par les taxations que firent les Bernois sur les biens immobiliers pour lever un impôt extraordinaire en faveur du rachat des hypothèques qui pesaient encore sur le Pays de Vaud.

Dans un article révélateur ${ }^{24}$ M. L. Junod donne des renseignements précieux sur la situation financière des habitants des paroisses de Lavaux et particulièrement sur celle

22 M. Reymond. Les origines de l'autonomie communale au Pays de Vaud. Extr. de la Revue historique vaudoise, 1928, page 29.

23 Plans généraux de la Paroisse de Lutry 17o5, folio 9, G II IX, 147 b.

24 L. Junod. Essai sur la propriété foncière à Lavaux en 1550. Mélanges offerts à M. P.-E. Martin. Genève 1961, page 255. 
de Lutry. On connaît le rapport entre le total des habitants non-possédants et le total des habitants, pour la commune et pour ses différentes régions. Même si le lieu de résidence de 10 non-possédants n'est pas connu, le tableau reste significatif:

$\begin{array}{lccc}\text { Possédants } & \text { Non-Possédants } & \begin{array}{c}\% \text { des Non-Possédants } \\ \text { par rapport au total } \\ \text { de la population }\end{array} \\ \text { Lutry } & 85 & 33 & 29 \\ \text { Savuit } & 12 & 3 & 20 \\ \text { Châtelard } & 7 & 2 & 22 \\ \text { Corsy } & 6 & 2 & 25 \\ \text { Montagny } & - & 1 & -37 \\ \text { Sur le Mont } & 25 & 15 & \end{array}$

Si l'on fait le rapport entre les non-possédants et les possédants on constate que le $39 \%$ de Lutry (33/85) est le plus bas pour le Lavaux si l'on met à part les $23 \%$ de Saint-Saphorin. Il faut voir là une conséquence de la sécularisation due au régime bernois. Dans les communes où les terres étaient en bonne partie entre les mains du clergé (c'était le cas pour Lutry et Saint-Saphorin) le rachat fut facilité.

Il nous reste maintenant à examiner de plus près deux autres chiffres et à essayer d'en tirer une signification sociologique. C'est le $29 \%$ de Lutry et le $37 \%$ de Sur le Mont. Ce sont les rapports les plus élevés de la commune. Si l'on considère celui de la ville même, on peut y trouver la confirmation de ce qui était avancé précédemment, à savoir qu'il y avait une société sans biens immobiliers qui ne vivait que de son travail et qui de ce fait ne pouvait être taxée. La présomption devient certitude lorsqu'on trouve des couturierstailleurs dans les non-possédants. Parmi les possédants on trouve d'une part de grosses fortunes, dont nous citons à dessein les noms

$\begin{array}{ll}\text { Provide Claude Gantyn } & 1433 \text { florins } \\ \text { Antoine et Jacques Sechaulx } & 1028 \text { florins } \\ \text { George Gabusat } & 1125 \text { florins }\end{array}$

il y en a quinze en tout et puis à l'autre extrémité 58 petits propriétaires possèdent moins de 500 florins. La fortune des autres se situe entre 500 et 1000 florins.

Le second rapport de 37\% nous montre une profonde différence du niveau de vie. C'est la seule région de la commune où les non-possédants sont aussi nombreux et il faut ajouter à cela que les plus hautes fortunes dépassent à peine les 200 florins. Il y a naturellement une différence énorme entre la valeur d'une pose de vigne à Lutry et une pose de terre à Savigny (rapport 1/10), cependant il s'agit de regarder de plus près l'origine de la population et son mode d'implantation, tout en relevant déjà la densité très faible dans cette contrée nord de la commune ( $22 \mathrm{~h}$. par $\left.\mathrm{km}^{2}\right)$.

\section{Les Monts de Lutry - pays de colonisation}

Sans pouvoir prouver déjà nos dires nous pensons qu'il faut faire intervenir deux facteurs dans le peuplement de cette contrée. Le premier de ceux-ci est la conquête progressive sur la forêt par les gens de Lutry même. Cette région était parfaitement complémentaire de celle du bord du lac. On y trouvait tous les bois comme on l'a déjà vu, on pouvait y aménager de nouveaux pâturages, cultiver ce qu'on avait supprimé en bas pour faire place à la vigne (céréales). Peu à peu les bourgeois les plus aisés allaient acquérir des terres sur les Monts en les rachetant après la sécularisation et construire des abris pour le bétail, des remises pour le foin et la paille comme nous l'indiquent encore les noms de maisons ou de propriétés actuelles; le Casard qui vient de case, les différentes Grangettes et les trois noms de lieux significatifs, la Gantène, la Séchaude, la Gabusat qui sont les noms des propriétés des trois plus riches habitants de Lutry cités ci-dessus. 
Une véritable économie agricole se développe, nécessaire pour ravitailler en viande, en céréales une population du bord du lac toujours plus nombreuse. Le second de ces facteurs est un afflux régulier de population étrangère. Sans avoir de données précises et annuelles on peut en consultant le livre d'or des familles vaudoises ${ }^{25}$ constater à partir du début du XVIe siècle une importante immigration. Depuis cette date et jusqu'à l'année 1862, 249 familles ont été reçues dans la commune de Lutry dont plus de cinquante viennent de Savoie ou de Haute-Savoie. La seule paroisse de Samoens en a fourni 8 .

Jusqu'à présent on a trop souvent considéré en Suisse les phénomènes d'immigration comme des conséquences aux mesures prises contre les protestants des pays voisins. De nombreux étrangers sont arrivés dans nos régions avant 1536 . Les causes de la migration des habitants du Faucigny sont connues. «Le Faucigny a possédé ses chaudronniers et ses maçons» 26 .

L'économie alpestre savoyarde ne permettait pas de nourrir la population autochtone, aussi une partie de celle-ci émigrait-elle quelques mois de l'année et allait offrir ses services à l'étranger.

Ces gens sont peut-être venus travailler au Lavaux pour la construction des murs et ont vu la possibilité de s'établir sur les Monts où l'agriculture leur paraissait plus rentable que chez eux. A moins d'avoir une grosse fortune ou un métier qui ne nécessitait pas d'achat de terrain, il devait être impossible pour eux d'habiter dans le vignoble, en revanche l'établissement sur les Monts comme fermier devait être facilité par les besoins de l'économie viticole, qui imposait de plus en plus le défrichement de grandes surfaces incultes à disposition. Le rapport entre les possédants et les non-possédants devient alors plus clair, l'exploitation des «Hauts» se faisait autrefois surtout dans l'intérêt de ceux du bas. La bourgeoisie de Lutry ainsi que l'afflux de population étrangère furent déterminants dans le mode de cette colonisation, qui engendrait un véritable prolétariat agricole.

\section{L'implantation dans le territoire de la commune}

On a déjà relevé les caractères urbains de la ville de Lutry (contiguité des bâtiments, fortifications, équipement collectif, société différenciée avec artisanat); il faut maintenant étudier l'implantation en se dirigeant vers les Monts. On est frappé lorsqu'on regarde avec attention le plan de 1821 par les formes très variées de l'habitat. Au bord du lac, Lutry constitue une cité agglomérée, entourée de vignes, presque totalement dépourvues de maisons éparses. Lorsqu'on s'élève du côté des Monts on rencontre entre les cotes 400 et $550 \mathrm{~m}$. une nouvelle forme d'habitat aggloméré, mais plus restreinte; ce sont les hameaux de Corsier-dessous, Corsier, Savuit, le Châtelard. Les vestiges néolithiques du Châtelard et ceux de Savuit d'origine romaine attestent leur ancienneté. Il faut peut-être voir dans le relief les causes de cette implantation.

Savuit se trouve à l'abri d'un affleurement de molasse dominant de haut le lac; le vieux Châtelard construit sur un bombement de même nature surveille la région, son nom d'ailleurs indique bien le caractère défensif de l'agglomération. Si des accidents topographiques ont engendré une forme d'habitat, d'autres facteurs d'ordre exclusivement humain et économique sont intervenus pour la développer.

La viticulture nécessite, nous l'avons vu, des travaux incessants, d'autre part la maind'œuvre employée est nombreuse, il faut donc, pour des raisons d'économie, se trouver le plus près possible des surfaces à cultiver. Ces impératifs seraient propres à engendrer un

25 H. Delédevant et $H$. Henrioud. Le livre d'or des familles vaudoises, Lausanne 1923, p. LXXIX-LXXXI.

$26 \mathrm{H}$. Onde. L'occupation humaine dans les grands massifs savoyards internes. Paris, Grenoble, 1942, page 323 . 
habitat dispersé si d'autres ne venaient pas en quelque sorte regrouper cette dispersion. En effet, la forte pente, la surface relativement restreinte à disposition de la vigne entraînent la concentration des maisons sur les replats et le long de chemins. L'habitat serré a, de plus, l'avantage d'offrir quelque fraîcheur durant l'été.

Si les facteurs économiques et humains ont joué pour l'habitat du vignoble, ils ont également joué pour celui que nous rencontrons après avoir franchi la limite de la vigne, qui est typiquement un habitat dispersé. J.Tricart ${ }^{27}$ a énuméré et analysé les causes d'un tel habitat. Pour le cas qui nous occupe, elles nous semblent avant tout économiques, politiques, éthniques.

Economiques d'abord parce que la colonisation fut la conséquence d'une transformation du système de culture de la région du bord du lac et qu'il a fallu trouver rapidement les moyens de nourrir une population tournée de plus en plus vers une culture commerciale.

Politiques ensuite parce que le mode de colonisation individuelle, par fermes isolées sur d'anciennes terres communales ou sécularisées, est étroitement lié à l'avènement de la bourgeoisie capitaliste et au système de faire-valoir indirect.

Ethniques peut-être enfin parce que, quoiqu'en majorité de Savoie la diversité de l'origine des immigrants (Piémont, Languedoc, Massif Central) ne devait pas les pousser instinctivement à une vie communautaire très étroite.

Il faudrait citer d'autres causes encore comme la vocation de la région qui est l'élevage et qui entraînait les «enclosures». Nous sommes presque certain que les exploitations étaient entourées de haies sur le territoire de Savigny. Un article, tiré du livre des conditions de la commune de Savigny de $1833^{28}$, s'adresse au voyer en ces termes: «Le voyer aura soin de faire émonder les haies, qui bordent les chemins publics, par les particuliers propriétaires, il veillera à ce qu'aucun empiètement ne se fasse au détriment de la largeur que doivent avoir les chemins en raison des bornes plantées.» Il nous reste à expliquer le ou les facteurs qui ont déterminé l'emplacement des maisons. Les habitants actuels prétendent que les maisons se sont établies autour de points d'eau. Il est naturellement très difficile dans cette dispersion originelle sur un terroir de mise en valeur progressive de savoir si la dispersion fut anarchique, sans sites attractifs déterminants ou si la dispersion fut organisée, chaque ferme disposant de tous les avantages qu'offre la région. Le dessin des parcelles formant un manteau d'arlequin fait plutôt penser à la dispersion anarchique (voir fig. 4). Il y a cependant une constatation que l'on doit faire, c'est la proximité des maisons isolées le long des chemins principaux. Nous avions relevé dans la première partie du travail des dépôts glaciaires dans la région de Savigny; ils occupent une grande surface et engendrent à cause de leur nature argileuse des régions marécageuses (les Mollies, la Goille). Les paysans de Savigny nous ont dit consacrer annuellement des chars de gravier à leurs chemins, la charge disparaissant très vite, absorbée par des sols mous. Il dut certainement y avoir des difficultés de transport à l'époque et on tenait ainsi à être à la fois près de sa surface cultivable et en bordure d'un chemin, ce qui facilitait les déplacements (transports de bois, de paille, de fumier avec la région du bord du lac). L'habitat dispersé le long des chemins semble mettre en évidence ce qu'on pourrait appeler l'axe de colonisation. En effet, presque tous les chemins sont orientés selon un axe Sud-Ouest - Nord-Est jusqu'en bordure du bois du Grand-Jorat contre lequel la colonisation est venue comme butter. On peut dès lors affirmer sans trop de risques que la colonisation fut conditionnée par Lutry car aucun centre à l'intérieur du Jorat ne pouvait motiver une extension pareille de l'habitat.

27 J. Tricart. L'habitat rural, C. D. U., Paris (s. d.), page 9o et ss.

28 Archives communales de Savigny (non classées). 


\section{LA DOMINATION BERNOISE, PÉRIODE DE RICHESSE POUR LE VIGNOBLE}

Si l'on regarde le plan de Lutry de 1705 on s'aperçoit que la vigne occupe à peu près la même superficie que de nos jours. L'extension maximum du vignoble dès le XVIIIe siècle est la conséquence d'une politique du vin amorcée par le clergé et reprise par l'aristocratie bernoise. Malgré les difficultés $\because 9$ qui apparurent dans la commune à l'arrivée des Bernois, ces derniers décidèrent de ratifier la conservation des droits, des franchises et libertés, écrites et non écrites des citoyens de Lutry ${ }^{30}$. Les Bernois donnèrent à la ville en 1539:1) Les biens d'une grande confrérie composée de quatre ou cinq plus petites qui avaient été jointes; 2) les fonds et rentes du petit monastère de Savigny au Jorat; 3) les biens de la chapelle de la Trinité. Quelques années après l'arrivée des Bernois, la ville de Lutry voit donc son pouvoir renforcé, le nombre des ses vignerons-propriétaires augmenter. Le rattachement de notre pays au Canton de Berne eut encore une autre conséquence, celle de lui créer un nouveau débouché pour ses vins. L'importance du pressoir et de la cave de LL.EE. sur le plan de 1705 en est un témoignage. Vers le milieu du XVIIe siècle la perspective du gain était si grande que «nombre de propriétaires vaudois surtout dans la vallée de l'Orbe et dans la région de Saint-Prex avaient transformé leurs champs en vignes.» Non seulement la vigne devenait intéressante, mais encore l'imposition sur cette nouvelle culture était modique. Ces vignerons de fraîche date ne payaient en effet que la dîme des novalles, très faible, parce qu'elle avait été créée de toute ancienneté pour favoriser les défrichements ${ }^{31}$. Le gouvernement dut en 1663 interdire toute transformation de champs en vignes sous peine d'extirpation des ceps et d'une amende de 100 florins par pose afin de protéger les vignobles anciens. Des rapports de l'automne 1672 constatent que depuis ces neuf dernières années, on a planté 136 poses de vignes nouvelles et 46 poses d'auttins. La même année l'ordre est donné d'extirper dans les trois semaines les vignes plantées depuis 1663 avec ordre aux baillis de faire rapport immédiat sur l'exécution.

«Le 5 décembre 1674 le gouvernement bernois défend catégoriquement d'acheter du vin savoyard, genevois, français ou bourguignon dans le pays ou en dehors, d'en amener, d'en trafiquer au dehors, d'en transporter par le pays à dos de cheval ou par chars. Les ordres nouveaux firent effet pendant quelque temps, puis on les oublie. En 1723 on constate que depuis la dernière interdiction, soit depuis 12 ans le vignoble vaudois a sérieusement augmenté et particulièrement entre Lausanne et Villette où 120 poses de vigne ont été plantées.» ${ }^{32}$

Un des signes extérieures de la haute conjoncture économique a toujours été la construction, or c'est à l'époque bernoise que la ville de Lutry a pris son caractère définitif, que la maison vigneronne, que celle des Monts également affirmèrent leur type.

A Lutry et dans les hameaux environnants la maison doit répondre à différents impératifs qui découlent directement de la culture de la vigne. Peu à peu devant l'augmentation régulière des récoltes, les vignerons ont commencé à construire leur propre pressoir. Il occupe d'habitude avec la cave tout le rez-de-chaussée. Cette dernière se trouve à côté du pressoir ou derrière lui, creusée déjà dans la pente où la température est plus fraîche. On y met les tonneaux, tandis que le matériel pour la vigne, relativement restreint, prend place aisément dans le pressoir. Le premier étage est généralement réservé à l'appartement du vigneron tandis que l'étage supérieur comporte une ou deux chambres à la disposition de la main-d'œuvre supplémentaire qui arrive à l'occasion des effeuilles et des vendanges. Au-dessus se trouve le «galetas» dans lequel on met le bois, la paille, le foin qu'on monte par l'extérieur grâce à une poulie et qu'on entre par une porte ouverte

29 Dictionnaire historique géographique et statistique du canton de Vaud. Tome I-II. E. Mottaz, Lausanne, F. Rouge, 1914-1921, page 173.

30 detto, page 174.

31 M. Reymond. Le vignoble vaudois à travers les siècles. Lausanne 1933, page 22.

32 M. Reymond. Le vignoble vaudois à travers les siècles. Lausanne 1933, page 24. 
dans le toit et abritée elle-même par un toit en mansarde. Cette construction se nomme le dôme ou «la quetale».

Il est rare que les vignerons n'aient été que vignerons. Avant l'apparition du phylloxéra qui a exigé une vigilance et un soin beaucoup plus soutenus, le vigneron possédait encore quelques têtes de petit bétail sinon des vaches. On trouve encore à Lutry des étables à cochons désaffectées. Cette ville abritait un troupeau jusque vers 1925-1930, le Châtelard jusqu'en 1936. Si Savuit ne possède aujourd'hui plus de porcs, cette agglomération a cependant gardé une vache.

Cette double activité entraînait naturellement des modifications dans la construction. Un vigneron ne pressait pas son raisin mais encavait seulement «son boire», il consacrait un peu de place à sa cave, réservant le reste du rez-de-chaussée à son étable et ses «boitons»; un autre vigneron, plus gros propriétaire de vignes, plaçait ses vaches sur les Monts et ne gardait en bas que porcs et moutons. Comme pour le caractère de l'agglomération, il y a pour celui de la maison un dégradé: plus on est près du lac, plus les traits de la maison vigneronne sont affirmés, plus on s'en éloigne, plus ceux de la maison paysanne sont marqués.

L'orientation de la maison est indifférente dans le vignoble. Placées en ordre contigu, les maisons se suivent perpendiculaires ou parallèles au chemin. Il n'en est pas de même dans l'habitat dispersé de Savigny ; si les maisons sont généralement placées à proximité d'un chemin, elles sont presque toutes orientées conformément à l'axe des vents dominants, à savoir selon l'axe Sud-Ouest - Nord-Est. L'altitude, la prédominance des courants froids ont une influence prépondérante sur l'orientation de la maison. Par économie on a placé tous les locaux sous le même toit, l'étable dégage une certaine chaleur dont peuvent profiter les chambres d'habitation, d'autre part la remise à bois sert de première protection contre les attaques de la bise. On a donc en général une façade nord-est constituée par le hangar à bois; le corps central formé de l'étable et l'appartement compose la section sud-ouest. Nous avons rencontré une anomalie où l'habitation était entre la remise à bois et l'étable, l'agrandissement postérieur a promptement remis les choses dans un ordre rationnel et l'habitat premier est aujourd'hui abandonné à la basse-cour. L'action du froid et des intempéries conditionne presque toutes les propriétés de la maison du Jorat. Généralement basse (un étage sur rez) la maison se protège le plus possible. Même la façade exposée pourtant au Sud-Est comporte un revêtement partiel, en éternite de nos jours, en bois autrefois, qu'on nomme la chape, et qui protège la construction contre les rafales de vent accompagnées de pluie, de neige ou encore de grêle.

Sur toute l'étendue de la chape la percée des fenêtres est presque exclue, afin de donner la plus grande efficacité à ce bouclier, et de ne pas l'endommager lui-même. Le côté sud-est est réservé à la percée des portes et fenêtres, tandis que le côté nord-ouest adossé souvent à la pente est flanqué d'un élément perpendiculaire de bois formé essentiellement d'une grande porte avancée, couverte d'un toit, qui permet ainsi de parvenir par derrière à la grange directement et d'éviter de monter le foin et la paille au prix de pénibles efforts. Relevons encore la fréquence des «neveaux» cités par Ch. Biermann ${ }^{33}$; presque chaque ferme à Savigny possède cette place abritée, pour que le paysan puisse y travailler. Elle existe simplement du fait que l'étable a été construite un peu en retrait de la remise à bois et des chambres d'habitation.

A Lutry comme à Savigny les matériaux employés pour la construction sont les mêmes; la parenté du sous-sol, en entraîne une nouvelle. Les argiles déposées par les glaciers permettent très tôt déjà la création d'une «Tuilière» à Savigny; les graviers encore exploités de nos jours à la Claie-aux-Moines, les galets des différents cours d'eau (Lutrive, Bussy) ont fourni les éléments d'un mortier solide, tandis que la molasse friable et poreuse, mais facile à tailler, servait pour les chaînes d'angle, les encadrements 
des fenêtres et des portes, les corniches, les arcades des portes de grange ou de pressoir ${ }^{34}$.

$34 \mathrm{Ch}$. Biermann. La maison paysanne vaudoise, Lausanne, Librairie de l'Université. F. Rouge, 1949 , page 37.

\section{V. ÉCONOMIE ET POLITIQUE}

\section{La séparation des deux communes et l'importance des bois}

La prospérité économique dans le vignoble durant la période bernoise n'avait eu pour effet que d'accentuer la différence de niveau de vie entre les deux régions de la commune. L'esprit de la Révolution vaudoise permit aux habitants des «Hauts» de présenter une requête intitulée "Pétition pour obtenir l'érection de Savigny en commune dinstincte et indépendante» ${ }^{35}$. Les divergences fondamentales qu'on peut relever dans ce manifeste reposent sur 1) la distance qui sépare les habitants du haut de ceux du bas, 2) la différence de nature des agglomérations et celle de l'esprit des habitants. La distance empêche les citoyens de Savigny de prendre une part active à la vie politique de la commune et les fastes de la ville de Lutry excédaient la population généralement pauvre de Savigny. On peut lire dans la pétition que rien ne manque à Lutry, ni bâtiments publics, ni médecins, ni sage-femme, ni organiste, ni fonteniers, ni conducteurs d'horloges, ni four banal, ni pavé, ni promenade. Lutry semble engloutir la plupart des revenus communaux et Savigny se plaint de n'avoir que 350 francs, quatre toises de bois de sapin, le produit de cinq poses de terrain pour entretenir deux collèges, en construire un nouveau et participer à la moitié des frais d'entretien du temple. Les auteurs du manifeste ne se contentent pas seulement de demander la séparation, mais ils préconisent déjà un programme économique afin de sortir la région de sa passivité artisanale. Ils prévoient des établissements de charronnages, le travail des futailles, le travail sur bois, la confection de paniers, de corbeilles, de vans, le tressage de la paille. Les communiers mettent enfin l'accent sur le revenu des forêts qui presque toutes leur reviennent si la séparation à lieu d'après les limites paroissiales.

La réponse des bourgeois de Lutry intitulée «Mémoire d'Information de la commune de Lutry au Grand-Conseil du canton de Vaud, sur la demande des Monts de Savigny d'être séparés de cette commune pour former une commune différente» ${ }^{36}$ n'a pas à être analysée dans ce travail.

Il suffit de citer un passage pour démontrer le caractère spécieux de leur argumentation.

«Les revenus, on le répète, ne sont point distribuables proportionnellement à l'étendue du territoire, ou au nombre de ses habitants, mais selon l'exigence et les circonstances des diverses localités de la commune.» ${ }^{37}$

En fait, les habitants de Lutry redoutent la perte de leurs forêts et ils veulent l'éviter à tout prix.

Lorsque le canton décida la séparation des deux communes d'après les limites paroissiales il fallut de longues palabres pour faire le partage des bois. Il se fit sur la base du nombre des bourgeois de chaque nouvelle commune. Lutry obtenait ainsi les $4 / 5$ des forêts. Ces forêts n'étaient d'ailleurs pas toutes de même nature et certaines étaient plus convoitées que d'autres (voir fig. 1).

sur Savigny

le Grand Jorat, bois de sapin

le Crêt des Côtes, jeunes sapins et chênes

le bois de Nyalin, hêtres

le bois de Burquinet, hêtres

$\begin{array}{rrc}\text { poses } & \text { toises } & \text { ha } 38 \\ 586 & 69 & 263,7 \\ 24 & 373 & 11,1 \\ 9 & 263 & 4,27 \\ 11 & 375 & 5,26\end{array}$

35, 36 Archives cantonales vaudoises, K XVIa 5.

37 Archives cantonales vaudoises, K XVIa 5, page 27.

38 Archives cantonales vaudoises, K XVIa 5, rapport du 2o novembre 1824. 
sur Lutry

le bois de la Ville, sapins $\quad 152$

le bois de la Gamaire, sapins

le bois des Dailles, chênes, sapins et pins

Si l'on regarde leurs essences, on s'aperçoit que les sapins prédominent sur les feuillus, les bois de chênes vont donc être âprement disputés, Savigny veut le Crêt des Côtes, car le chêne lui est indispensable pour les ponts et la construction de ses établissements publics. Lutry avide de bois de chêne pour ravitailler ses tanneurs et ses tonneliers, n'est pas disposée à abandonner cette réserve qu'elle considère comme personnelle. En outre d'après le partage, le Bois du Grand Jorat, le plus important en étendue, un des plus beaux bois du canton se trouvera sur la commune de Savigny.

Finalement après de multiples tractations, Lutry réussit à obtenir sur son territoire le Bois de la Ville, le Bois de la Gamaire, le bois des Dailles, le Bois-de-la Chaux et sur le territoire de Savigny la partie orientale du Bois du Grand Jorat, le bois de Burquinet et celui du Crêt-des-Côtes, tandis que Savigny se retrouvait avec la partie occidentale du bois du Grand Jorat, le ruisseau de la Craivaveyre faisant frontière, plus le bois de Nyalin. A l'issue de ce partage on ne s'étonne pas qu'un certain ressentiment ait subsisté chez les habitants des Hauts.

Savigny comprit rapidement l'importance de ses bois; aussi ses autorités nouvellement élues s'efforcèrent-elles de frapper très lourdement les déprédateurs fort nombreux à cette époque.

Aujourd'hui encore pour les deux communes, les bois sont d'appréciables sources de revenus. La forêt productive prend de l'extension; elle passe en 18 ans de 231 à 237 hectares. Le volume total passe de $69524 \mathrm{~m}^{3}$ à $78484 \mathrm{~m}^{3}$ et le volume par ha. augmente également alors que le nombre de plantes diminue. Le rendement à l'hectare a passé de $1318 \mathrm{~m}^{3}$ à $1884 \mathrm{~m}^{3}$. Relevons enfin l'effort de reboisement, porté surtout sur les feuillus puisqu'en 18 ans les résineux ont augmenté de $10 \%$ alors que les feuillus enregistrent une augmentation de plus de $90 \%$.

\section{L'aménagement définitif du vignoble et les variations de son étendue}

C'est vers la fin du XVIIIe siècle et le début du XIXe que le vignoble prit son aspect définitif. Dans chaque contrat l'entretien des murs et coulisses fait l'objet d'un article important. Sous l'influence des sociétés d'agronomie et surtout sous celle de la Confrérie des vignerons les soins à porter à la vigne sont de plus en plus l'objet d'études scientifiques. Dans un contrat passé entre la commune de Savigny et son vigneron, on peut notamment lire à l'article 2: «Le vigneron ne devra planter, ni ne sèmera aucun jardinage de quelle espèce que ce soit dans aucune des vignes appartenant à la commune» ${ }^{39}$. Il est intéressant à ce propos de lire les recommandations de M. Brun-Chappuis: «Au commencement de mon établissement, ne connaissant pas mes véritables intérêts, ni la culture du raisin, je recoltais annuellement sur mes vignes environ de 5 à 7 sacs de blé, gros blé, ou maïs, un ou deux de fèves et un de haricots; depuis quelques années, je n'en plante pas un grain. Cela dit assez combien je me suis convaincu que l'introduction de toute espèce de légumes était préjudiciable à la vigne.» ${ }^{\mathbf{4 0}}$ Non seulement les cultures intercalaires disparaissent mais encore les arbres. Dans ce même contrat ${ }^{41}$ on peut lire à l'article 14: «Le vigneron ne plantera aucun arbre, dans quelle vigne que ce soit, et ceux qui y existent maintenant devront être arrachés au premier ordre qui sera

39 Livre des conditions de la commune de Savigny de 1833.

40 M. Brun-Chappuis, Traité sur la culture de la vigne telle qu'elle se pratique à La Vaux, Genève 1824, page 29 .

41 Livre des conditions de la commune de Savigny de 1833. 
donné.» A cette époque déjà on pratiquait la taille encore en usage aujourd'hui, c'est la taille vaudoise ou taille de la confrérie, généralement connue sous le nom de taille en gobelet. Elle est courte sur charpente courte ${ }^{42}$. Sur ce point aussi les contrats sont clairs, la teneur de l'article 16 est la suivante ${ }^{43}$ : «Le vigneron ne pourra surtailler (soit métailler) ni arracher la vigne sans en avoir prévenu la Municipalité.» Cultures à éliminer de la vigne, soins à porter à la terre, aux murs, à la plante elle-même, choix des plants suivant le terroir, tout est recommandé. «Rien n'est mieux planté, dressé, échalassé, aligné, épampré, rogné, labouré, sarclé et fumé qu'une vigne suisse au bord du Léman.»44

Ce contrat tiré du «Livre des conditions de la commune de Savigny» de 1833 ne précise pas les plants des vignes de la commune, cependant à l'article 9 il est dit ${ }^{45}$ : «Il est expressément défendu au vigneron, d'introduire du rouge dans les vignes soumises à sa culture, les ceps de rouge qui pourraient se trouver mêlés avec les ceps de blanc seront détruits de suite, et quant aux places qui seront complètement en ceps de raisins rouges, ils les détruiront pour les réintroduire en ceps de raisins blancs, à la réquisition qui leur en sera faite par la Municipalité.»

Le libellé de cet article prouve qu'on produisait presque exclusivement du vin blanc. Il semble bien qu'il en ait toujours été ainsi, Madame Courtieu-Capt écrit: «Ils (les actes) ne disent jamais quelle est la couleur du vin vendu, à de très rares exceptions: lorsqu'ils en parlent, c'est toujours de vin blanc dont il s'agit ...»46. Si l'on compare les productions de rouge à celles de blanc on s'aperçoit que les premières représentent à la fin du XIXe siècle $5 \%$ seulement des secondes.

Récoltes à Lutry 47

$\begin{array}{ccc} & \text { Rouges hl } & \text { Blancs hl } \\ 1891 & 222 & 4416 \\ 1892 & 270 & 17 \text { o } 28 \\ 1893 & 800 & 28200 \\ 1894 & 576 & 20304 \\ 1895 & 360 & 12690 \\ 1896 & 468 & 15228 \\ 1897 & 440 & 12650 \\ 1898 & 400 & 10626 \\ 1899 & 240 & 7590 \\ 1900 & 720 & 22770 \\ 1901 & 680 & 21505\end{array}$

Si nous prenons des chiffres plus récents nous constatons que le rapport est sensiblement le même.

\begin{tabular}{|c|c|c|c|}
\hline \multirow[b]{2}{*}{1938} & \multirow{2}{*}{$\begin{array}{c}\text { Blancs européens } \\
2713,80\end{array}$} & \multicolumn{2}{|l|}{ Rouges producteurs } \\
\hline & & 39,50 & \\
\hline & & $\begin{array}{c}\text { Rouges européens } \\
\mathrm{hl}\end{array}$ & $\begin{array}{l}\text { Rouges européens } \\
\text { directs } \mathrm{hl}\end{array}$ \\
\hline 1945 & 9536 & 260,91 & 191,15 \\
\hline 1951 & 12608,40 & 1540 & 420 \\
\hline 1956 & 3410 & 57,20 & 140 \\
\hline 1960 & 22785 & 88 & 270 \\
\hline
\end{tabular}

On peut se demander la cause de cette persistance des vins blancs alors que de nos jours l'on sait que la majorité des buveurs de vin achète du vin rouge. Il y a, bien enten-

42 J. Dubois. Le vigneron vaudois et ses vins, comment il pourra en améliorer la vente, Lausanne, 1944.

43 Livre des conditions de la commune de Savigny de 1833.

44 J. Guyot. Etude sur les vignobles de France. Paris, 1863.

45 Livre des conditions de la commune de Savigny de 1833.

46 A.-M. Courtieu-Capt. Recherches sur l'histoire du vignoble au moyen âge dans la partie méridionale de l'ancien diocèse de Lausanne. Paris, Ecole des Chartes, 1959. (Epreuve polycopiée des Archives cantonales vaudoises).

47 Statistique agricole du canton de Vaud. 
du, des raisons économiques. Le travail qu'exige la vigne chez nous, à Lutry en tout cas, implique un certain rendement à l'ouvrier ou au fossorier $\left(450 \mathrm{~m}^{2}\right)$. On compte sur 400 litres. Le chasselas peut offrir ces rendements tout en produisant un vin qui reste de qualité. Si l'on voulait produire du vin rouge, il faudrait prendre des plants choisis car nous n'arriverions pas à concurrencer la production courante d'Espagne, d'Algérie ou encore d'Italie. Or il s'avère que le rendement d'un pinot noir n'est que de $300 \mathrm{l}$. au fossorier, alors que le Gamay déjà moins coté en produit à peine $50 \mathrm{l}$. de plus. On comprend mieux pourquoi les vignerons sont si réticents devant une transformation qui les amènerait à vendre une bouteille à un prix trop élevé.

Les vignerons de Lutry aiment avoir quelques parchets de plants du Rhin quoique leur rendement soit de $300 \mathrm{l}$. au fossorier. Ce plant qui demande davantage de soins donne un raisin plus doux qui améliore d'une manière sensible la qualité du vin.

L'extension ou la régression du vignoble vaudois dépend de quatre facteurs bien déterminés :

1) Obligation de pratiquer la conversion des cultures.

2) Possibilité d'écoulement; politique de la Confédération relative à l'importation de vins étrangers.

3 ) Disponibilité ou manque de main-d'œuvre.

4) Bonnes ou mauvaises récoltes.

Nous avons vu (page 71) un cas ancien de conversion obligée des cultures; il y a quelques années en compensation à une politique de soutien aux vignerons, la Confédération classa le vignoble suisse d'après la qualité de ses produits en 3 zones $(A, B, C)$. Seules pouvaient subsister les vignes situées en zones $A$ et $B$. Cette classification a une valeur secondaire pour notre commune, où il n'a pas existé, à notre connaissance, de zone C.

Pendant le XIXe siècle la production n'a cessé d'augmenter. «Le produit annuel du vignoble vaudois a passé de 43000 chars entre 1818 et 1832 à 73000 chars entre 1832 et 1837. L'exportation qui était de 18000 chars de 1818 à 1822 a passé à 25000 chars de 1832 à $1837 \gg 48$. En 1891 un rapide accroissement des importations força la Confédération à prendre des mesures pour protéger la production indigène. Elle décida une taxe sur les entrées de vins étrangers, prit en charge des surplus de production, porta secours aux vignerons dont les vignes a vaient été gelées en 1925 , 1933 et 1938 dans le canton de Vaud et organisa la vente de vin d'action de 1935 jusqu'en 1937.49 Plus récemment encore en 1956 l'Etat de Vaud est venu en aide aux victimes du gel à raison de

$$
\begin{aligned}
& 6 \mathrm{fr} \text {. l'are pour } 40 \text { à } 49 \% \text { de dégât } \\
& 10 \mathrm{fr} \text {. l'are pour 5o à } 69 \% \text { de dégât } \\
& 20 \mathrm{fr} \text {. l'are pour } 70 \text { à } 79 \% \text { de dégât } \\
& 40 \mathrm{fr} \text {. l'are pour } 80 \text { à } 100 \% \text { de dégât } 50
\end{aligned}
$$

En automne 1960 enfin, la Confédération a promis le rachat des vins excédentaires au printemps 1961, suivant les prix indicatifs, tout en avanøant immédiatement aux vignerons, l'argent garanti par le vin pris en charge. Les vignerons vaudois, en particulier, ne peuvent actuellement pas se plaindre de l'aide qui leur est apportée soit par les autorités cantonales, soit par les autorités fédérales.

Superficie du vignoble pour la commune de Lutry en ha.

$\begin{array}{llll}1887 & 261 & 1893 & 261 \\ 1888 & 261 & 1894 & 261 \\ 1889 & 261 & 1895 & 261 \\ 1890 & 261 & 1896 & 261 \\ 1891 & 261 & 1897 & 261 \\ 1892 & 261 & 1898 & 261\end{array}$

48 H. Rieben, J.-P. Gonvers et Ch. Iffland. Le canton de Vaud à la croisée des chemins. Lausanne 1962, page 17.

$49 \mathrm{~J}$. Dubois. Le vigneron vaudois et ses vins, comment il pourra améliorer la vente. Lausanne, 1944.

50 Document du Département de l'agriculture, de l'industrie et du commerce, service de la viticulture et de l'assurance-grêle. 1956. 


\begin{tabular}{|c|c|c|c|c|c|}
\hline 1899 & 261 & & 1914 & 240 & mauvaise année \\
\hline 1900 & 261 & & 1915 & $2+0$ & année moyenne \\
\hline 19o1 & 261 & & 1916 & $2+o$ & année moyenne à mauvaise \\
\hline $19 \circ 2$ & 261 & & 1917 & 200 & mauvaise année \\
\hline 1903 & 261 & mauvaise année & 1918 & 190 & bonne année \\
\hline 1904 & 260 & & 1919 & 215 & bonne année \\
\hline 1905 & 260 & & 1950 & 177 & 212 \\
\hline 1906 & 260 & bonne année & 1951 & 176 & 102 \\
\hline 1907 & 260 & mauvaise année & 1952 & 176 & $46+$ \\
\hline \multirow[t]{4}{*}{1908} & 260 & année moyenne & 1953 & 175 & 482 \\
\hline & & financement de la Confédéra- & 1954 & 176 & 100 \\
\hline & & tion et de l'Etat pour la recons- & 1955 & 175 & 585 \\
\hline & & titution du vignoble. & 1956 & 174 & 145 \\
\hline 1909 & 260 & mauvaise année & 1957 & 172 & 511 \\
\hline 1910 & 250 & mauvaise année & 1958 & 171 & 024 \\
\hline 1911 & 250 & relativement bonne année & 1959 & 169 & 838 \\
\hline 1912 & 250 & mauvaise année & 1960 & 166 & 522 \\
\hline 1913 & 245 & mauvaise année & 1961 & 165 & 47551 \\
\hline
\end{tabular}

Malgré la politique d'assistance au vignoble, on constate en regardant les chiffres de sa superficie une diminution constante. Il y a cependant au cours de cette diminution des phases de caractère très différent; d'une part des sauts brusques comme entre les années 1910-1919 et d'autre part une régression régulière comme depuis 1950 à nos jours.

La crise des années 1910 à 1919 relève de plusieurs facteurs. La vigne depuis l'apparition du phylloxéra demande des soins nouveaux. Les porte-greffes américains sont moins résistants aux maladies cryptogamiques; le nombre des sulfatages augmente. Malgré l'aide de la Confédération pour la reconstitution du vignoble, celui-ci régresse; le paysan-vigneron a disparu, il a remis sa terre à un vigneron qui tient à le rester, mais ce dernier a déjà de la peine à trouver de la maind'œuvre. En 1910 on peut lire dans le rapport du préfet Corboz «la difficulté de trouver des ouvriers devient toujours plus grande; les jeunes gens abandonnent la culture de la vigne. Les vieux qui ne peuvent pas se créer une nouvelle position restent». 52

Durant cette période, où des problèmes de main-d'œuvre se posaient les mauvaises récoltes se succédèrent pendant cinq ans. En 1917 dans son rapport de fin d'année le préfet Foscale écrit qu'il a grêlé pendant les vendanges, que la moyenne par récolte fut de 70 litres par fossorier. Découragés les vignerons de Lutry ont arraché 38 ha. Et le préfet d'écrire: «Reverra-t-on à Lutry le troupeau bovin qui existait encore il y a to ans?» «La construction suit son cours, surtout entre la ligne de chemin de fer Lausanne-St-Maurice et le lac; dans un avenir prochain, les vignes actuelles seront remplacées par de jolies villas. De ce fait, les terrains ont plus que doublé de valeur».53

En effet, si le vignoble disparaît, c'est au profit de la construction. Le problème de la main-d'œuvre se pose aujourd'hui plus que jamais. Malgré les herbicides et les «atomiseurs», la main-d'œuvre étrangère, sur laquelle les vignerons avaient pu compter jusqu'ici, mais qui maintenant est absorbée par les autres secteurs (hôtellerie, bâtiment surtout) va faire défaut. L'impossibilité de mécaniser davantage l'exploitation du vignoble du Lavaux pousse les vignerons à réduire leur surface de culture, tout en s'assurant une retraite confortable. Les sociétés immobilières paient maintenant 120 francs le $\mathrm{m}^{2}$ sur la commune de Lutry. Manque de personnel, offres pour le terrain alléchantes contribuent en grande partie à cette régression progressive.

\section{Savigny - une vocation qui s'affirme et la fin d'une économie complémentaire}

Dans son livre sur le Jorat, Ch. Biermann dit: «Le Jorat voisin est la région complémentaire de Lavaux avec ses champs qui fournissent la paille d'attache, ses engrais animaux, particulièrement nécessaires à la vigne, ses bois où se fabriquent les échalas. Moins

51 Statistique agricole du canton de Vaud. Service de la viticulture.

52 Statistique agricole de 1910, Lausanne 1911. Revue économique, industrielle et commerciale du canton de Vaud. Rapport de M. Corboz, préfet du district de Lavaux, page 34.

53 Statistique agricole de 1917 et 1918. Revue économique industrielle et commerciale du canton de Vaud. Lausanne 1919, rapport de M. Foscale, préfet du district de Lavaux, pages 24 et 25. 
serrés qu'autrefois où Lavaux demandait encore au Jorat les céréales, les pommes de terre, la viande de boucherie, le lait, les liens qui unissent ces deux pays si différents ont résisté à la séparation de la partie montagneuse des anciennes paroisses d'avec la partie viticole et à leur constitution en commune autonome» ${ }^{55}$. Si ce fut le cas jusque vers les années 1940, cette situation de nos jours a totalement changé.

Lors de la scission des communes, la «peuplade de Savigny, presque sans civilisation, population de prolétaires, dont la très grande partie ne possède rien, ou ne possède pas de quoi se nourrir» ${ }^{56}$ pratiquait une polyculture à des fins vivrières. Nous avons pu tirer du Registre des Actes d'origine de 1841-1871 (sic) les renseignements suivants sur la nature des cultures et leur rendement : 5

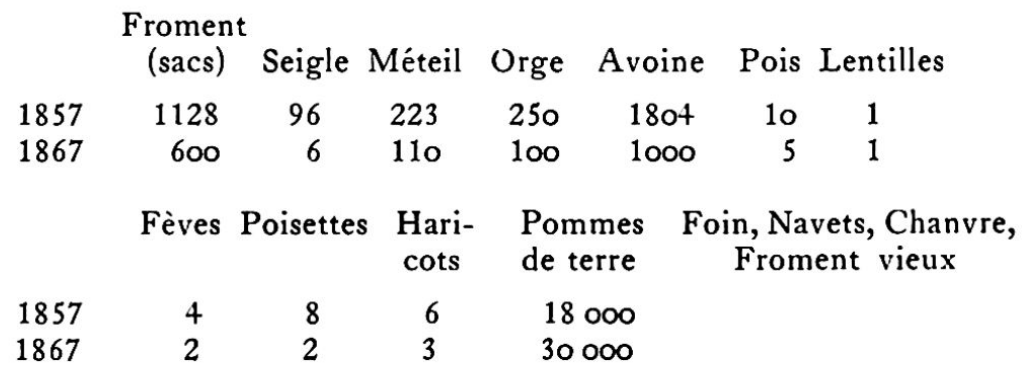

Variété des plantes cultivées et irrégularité des rendements sont les deux caractéristiques de la production agricole de Savigny. Non seulement le système de polyculture, mais encore le régime successoral familial d'alors traduisent la dépendance très stricte de la population de Savigny vis-à-vis de sa propre terre et de sa mise en valeur. Lorsqu'un paysan avait plusieurs fils, c'était le premier à se marier qui tacitement devenait le successeur, les autres pouvaient rester sur le domaine à condition de demeurer célibataires. Ceux qui se mariaient par la suite devaient quitter la ferme; on empêchait ainsi le morcellement des terres, on fournissait de la main-d'œuvre à bon compte, on freinait le mouvement démographique.

Cependant vers la fin du siècle dernier l'économie semble s'ouvrir un peu. Grâce à la création de l'industrie du chocolat au lait et des produits alimentaires à Vevey, la population put commencer à livrer du lait aux condenseries de cette localité. Peu à peu le cheptel devient plus grand, le climat, les prairies riches convenant très bien au bétail. La mise en service de la ligne Lausanne (Place du tunnel) - Savigny permit aux habitants d'écouler chaque jour leur lait dans la capitale; en 1905 la société laitière de Savigny, en 1928 celle de Mollies-Margot demandent leur adhésion à la Coopérative Laitière Lausannoise. Récemment celle du Martinet et de la Claie-aux-Moines ont décidé également de livrer leur lait à Lausanne. Bien des chiffres nous manquent quant à la production laitière de cette commune, cependant nous sommes parvenus à chiffrer la production pour 1950 et 1960 . Les résultats prouvent avec suffisamment de clarté l'augmentation de la production.

Production en $\mathrm{kg} . / \mathrm{l}$.

$\begin{array}{cccccc} & \text { Savigny } & \text { Mollie-Margot } & \begin{array}{c}\text { La Claie- } \\ \text { aux-Moines }\end{array} & \text { Le Martinet } & \text { Total } \\ 1950 & 844.364 & 298.090 & 506.088 & 144.709 & 1793.251 \\ 1960 & 1033.492,5 & 353.027 & 512.606 & 130.424,5 & 2029.550\end{array}$

54 Statistique agricole de 1908, Lausanne 1909. Revue économique, industrielle et commerciale du canton de Vaud. Rapport de M. Corboz, préfet du district de Lavaux.

55 Ch. Biermann. Le Jorat. Neuchâtel, Attinger, 191o, page 106.

56 Archives cantonales vaudoises K XVIa e. Mémoire d'information de la commune de Lutry au Grand-Conseil du canton de Vaud.

57 Archives communales de Savigny. Registre des Actes d'origine de 1841-1871. 
L'augmentation est patente, pourtant d'autres chiffres méritent notre attention. En effet, on s'aperçoit qu'à cette augmentation de la production correspond une diminution du nombre des vaches laitières. Les sociétaires de Savigny même possédaient en tout 382 vaches en 1950 alors qu'ils n'en possédent plus que 374 en 1960. Si l'on se penche sur les chiffres du troupeau de bovins pour toute la commune de Savigny, on peut faire la même constatation.

$\begin{array}{ccccc}\text { Nombre de bovins - Savigny } & & & \\ \text { bovins } & \begin{array}{c}\text { vaches } \\ \text { laitières }\end{array} & \begin{array}{c}\text { \% du } \\ \text { troupeau }\end{array} & \text { propriétaires } \\ 1939 & 1347 & 751 & 55,8 & \\ 1946 & 1120 & 590 & 52,6 & \\ 1950 & 1242 & 723 & 58,2 & \\ 1955 & 1271 & 715 & 56,3 & 129 \\ 1960 & 1496 & 770 & 51,5 & 119\end{array}$

Ainsi on remarque une augmentation du cheptel, mais en même temps une diminution du rapport vaches laitières/troupeau; c'est qu'à côté de la production de lait, se développe un élevage pour la boucherie. En 1915, le préfet Foscale note dans son rapport: «L'élevage du bétail bovin est en progrès à Savigny.» $58 \mathrm{La}$ culture herbagère convient plus que toute autre sous ce climat souvent rude, le rendement des céréales panifiables est faible $16,15 \mathrm{q} / \mathrm{ha}$., les betteraves à sucre poussent mal, il n'y a guère que les pommes de terre qui ont un rendement moyen $18000 \mathrm{~kg}$./ha. Superficie des céréales, superficie des pommes de terre reculent d'ailleurs au profit des cultures herbagères. Nous ne pouvons malheureusement nous fonder sur les chiffres donnés pour la superficie des cultures herbagères, les critères pour les estimations ayant été différents d'une année à l'autre; nous pouvons cependant relever le chiffre de $77,3 \%$, rapport élevé des cultures herbagères comparées au total des cultures. L'augmentation continue du troupeau a engendré plusieurs organismes, tel le syndicat d'élevage des Monts de Lavaux dont le siège est à Forel, ou tel le petit syndicat d'alpage privé, formé de 7 membres. Ces syndicats se chargent d'organiser la montée annuelle dans les Montagnes, aux Ormonts-Dessous, à la Croix de Javerne, à la Dent de Jaman ou encore dans la région de Châtel-Saint-Denis.

Le problème des fourrages a suscité la création d'un séchoir artificiel à Châtillens, dont une vingtaine des paysans de Savigny profitent. Toute cette spécialisation a fait qu'aujourd'hui le marchand de bétail de Savigny est le plus gros fournisseur de Lausanne.

Que reste-t-il alors du caractère complémentaire de cette région évoqué par Ch. Biermann? La disparition du seigle a forcé les vignerons à commander en Corrèze leur paille fine pour attacher la vigne, les échalas de bois sont en voie de disparition devant ceux en fer, et les derniers vignerons qui les utilisent les font venir de la région d'Ecotaux, éloignée de $30 \mathrm{~km}$., où les plantes sont moins cher à la mise et où la façon est meilleur marché. Les plantes des bois de Savigny sont bien trop belles, bien trop près de Lausanne pour qu'on puisse encore rentablement les débiter en échalas. Les tonneaux de chêne, eux, ont fait place aux vases de ciment.

Quant au lait, tout le trafic se fait maintenant par Lausanne. Aucun laitier de Lutry ne se ravitaille directement sur les Monts. De plus, la disparition des abattoirs de cette ville va certainement diminuer le commerce de bétail entre les deux régions, ce dont va profiter la capitale. Il ne reste guère que le fumier, élément indispensable à l'existence du vignoble, mais il n'y a guère que cela et cet état de fait montre bien le changement fondamental de l'économie de Savigny et des Hauts en général.

58 Statistique agricole de 1915, Lausanne 1916. Revue économique, industrielle et commerciale du canton de Vaud. Rapport de M. Corboz, préfet du district de Lavaux, page 26. 


\section{LES VOIES DE COMMUNICATION, FACTEUR DE DÉVELOPPEMENT}

Lutry se situait sur une artère qui était l'une des plus fréquentées dès l'Antiquité. Sa proximité avec le bord du lac permettait aux eaux parfois impétueuses de causer de graves dégâts qui rendaient les transports difficiles. Aussi les habitants riverains prirentils l'habitude de recourir au lac pour pratiquer le commerce puisque même après la réfection de la route datant de 1565 , cette dernière n'était pas meilleure «montant et descendant ${ }^{59}$

C'est la raison pour laquelle on peut voir sur le plan de Lutry de 1705 une galerie en prolongement sur le lac; il s'agit d'une construction en bois sur pilotis s'avançant dans le lac à dix m. environ, et à un mètre cinquante au-dessus du niveau de l'eau, de façon à permettre aux barques de charger et de décharger. Un toit recouvrait cette jetée de bois. Le port dut avoir une certaine importance puisque son aménagement moderne, jetées et débarcadère datent de 1836.

De nos jours le quai de Lutry prend encore de l'extension; grâce aux carrières de Meillerie, on a pu aménager un quai d'un $\mathrm{km}$. de long. Un service régulier de bateaux de la Compagnie générale de Navigation dessert Lutry en été.

La commune de Lutry dès septembre 1862 a le privilège de voir passer sur son territoire deux lignes de chemins de fer, celle de Berne et celle du Simplon, et de posséder deux gares, la sienne sur la ligne du Simplon, celles de la Conversion et la halte de Bossière sur celle de Berne. Bien placée sur la trajectoire des lignes les plus directes rayonnant depuis Lausanne, la commune de Lutry eut une grande chance car sa topographie se montrait plutôt hostile; la vallée profondément encaissée de la Borive (anciennement du ruisseau Bussy) nécessita la construction du pont qui porte ce nom, alors que le bombement molassique de Savuit-Bertholo obligea le percement d'un tunnel.

En 1896 un tramway reliait Lausanne à Lutry. Gênant passablement la circulation, il a été remplacé récemment par un autobus qui transporte les habitants de Lutry en moins de vingt minutes au centre de la capitale.

Si l'on examine maintenant la situation de Savigny dans le domaine des relations avec l'extérieur, le contraste est frappant. Savigny se trouve sur l'axe Lausanne-OronFribourg. Cependant ni l'une ni l'autre de ces deux dernières villes ne commerçaient beaucoup avec Lausanne. Le seul gros trafic à travers le Jorat s'écoulait par la route Lausanne-Montpreveyres-Moudon. En 1902 seulement, une ligne de tramway reliait Savigny à la Place du Tunnel. Aussi modeste fût-elle, cette ligne permettait de transporter assez rapidement et sans trop de frais la production laitière de la commune du Jorat; elle ne fut cependant jamais un stimulant pour le développement de Savigny. Aujourd'hui le tramway a vécu, peu rentable, fort coûteux, ce moyen de locomotion a fait place aux autobus.

Ainsi au début du siècle nous avons d'un côté une commune en relation directe avec ses environs et la capitale grâce à ses trois gares, son port, sa station terminus de tramway, ses deux routes parallèles au lac, l'une en bordure, l'autre sur les «Monts»avec deux perpendiculaires en direction de Savigny, et d'un autre côté une commune sauvée de l'isolement par une route de trafic secondaire et une transversale qui mène à Lutry ainsi que par une ligne de tramway en direction de Lausanne.

\section{ÉPOQUE ACTUELLE}

(voir fig. 6)

Démographie: L'opposition entre le dynamisme de la région du bord du lac et celle du Jorat va se trouver mise en évidence par les chiffres de population.

59 Ch. Biermann. Lavaux aus «Der Schweizer Geograph», 1935, page 10. 


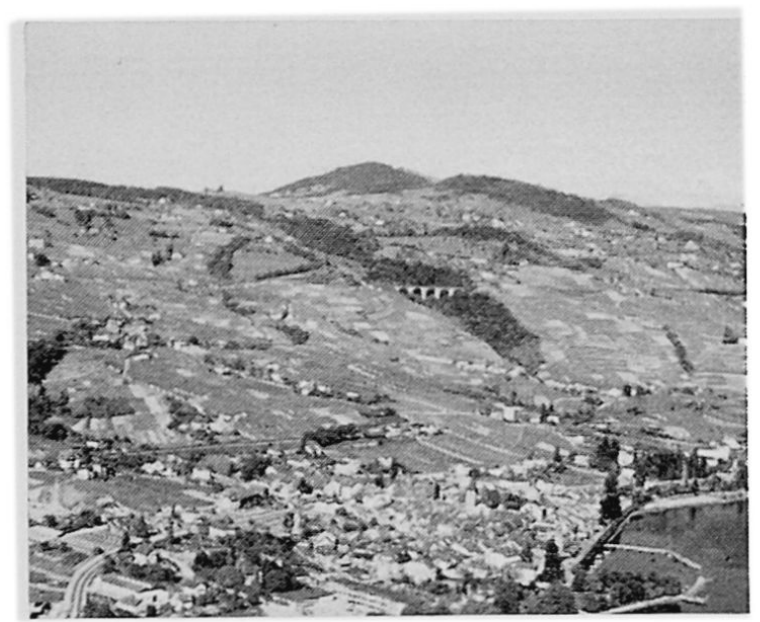

Figure 6

Sur cette topographie de coteau au bord d'un lac, l'antagonisme entre l'habitat et la culture de la vigne est puissant.

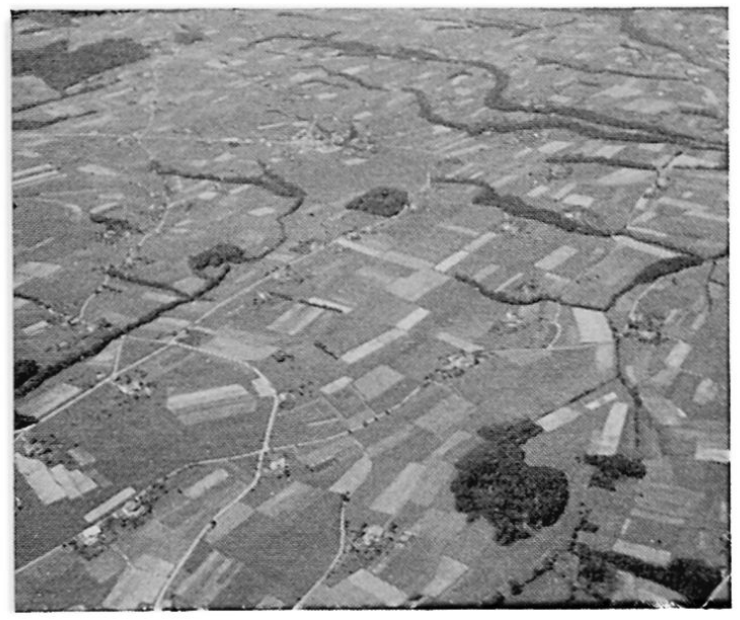

Le paysage tenant à la fois de l'openfield et du bocage l'irrégularité de la forme des champs la diversité de leur orientation, l'habitat dispersé enfin font de Savigny une commune d'une grande originalité

$\begin{array}{lrr} & \text { Lutry } & \text { Savigny } \\ 1707 & 1+87 & - \\ 1850 & 2011 & 10+2 \\ 1860 & 25+5 & 99+ \\ 1870 & 2185 & 1129 \\ 1880 & 22+2 & 11+1 \\ 1890 & - & - \\ 1900 & 22+3 & 1072 \\ 1908 & 2498 & - \\ 1910 & 2559 & 1088 \\ 1920 & 2+28 & 1031 \\ 1930 & 2595 & 978 \\ 19+1 & 2540 & 925 \\ 1950 & 2916 & 980 \\ 1960 & 3481 & 884\end{array}$

Nous voyons en effet une commune s'agrandir régulièrement, mis à part de courtes périodes de crise viticole, et l'autre au contraire se dépeupler tout aussi régulièrement. Savigny à cet égard occupe une situation marquante puisqu'elle se trouve à la frontière sud de la zone du plateau vaudois souffrant de dépeuplement.

L'analyse de la pyramide des âges nous révèlerait apparemment une structure identique pour les deux communes. Leur signification respective serait cependant tout différente. La présence d'un asile de vieillards à Lutry, des mouvements de population à sens inverse pour les deux agglomérations sont des éléments qui rendent l'interprétation plus difficile. Bien plus significatifs sont pour ces deux exemples les mouvements naturel et migratoire de la population.

Mouvement naturel et mouvement migratoire de la population

\section{Nombre des naissances par 1000 habitants}

$1941-1950 \quad 1951-1960$
Nombre des décès par 1000 habitants

$1941-1950 \quad 1951-1960$
Accroissement par excédent des naissances par 1000 hab. chiffr
Solde du compte émigrants/immigrants
chiffres absolus annuels 1941-1950 1951-1960

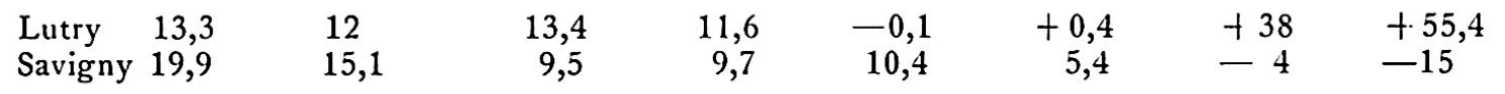


Flagrante différence, alors que Lutry durant les années 1941-50 a un taux de mortalité supérieur à celui de natalité, Savigny a un accroissement de 10,4 pendant la même période. C'est cependant Lutry qui s'accroît et Savigny qui régresse d'après les chiffres de la population. Exode rural allant en s'accentuant ( -4 habitants annuellement entre 1941-50 et -15 habitants annuellement entre 1951-60) pour l'une des communes et vocation de plus en plus marquée de banlieue résidentielle pour l'autre $(+38$ habitants annuellement entre 1941-50 et $+55,4$ habitants annuellement entre 1951-60). Ce sont les jeunes qui abandonnent Savigny, ce qui explique la chute très forte du taux de natalité encore de 10,4 entre 1941-50 à 5,4 pour la période 1951-60. Quant à Lutry le taux natalité reste bas malgré l'apport de la population extérieure. Cette situation s'explique par le fait 1) que la population vigneronne s'éteint lentement, 2) par le caractère résidentiel de la banlieue qui abrite une catégorie de gens aisés, venant se fixer plutôt tardivement et 3) par l'importance artificielle de la population agée due à la présence de l'asile de vieillards.

\section{Répartition de la population active selon les grands groupes d'activité}

\begin{tabular}{llllllllll} 
& \multicolumn{4}{c}{ Agriculture } & \multicolumn{4}{c}{ Secondaire } \\
& \multicolumn{1}{c}{ Artisanat, industrie } & \multicolumn{3}{c}{ tertiaire } \\
& 1930 & 1950 & $1960^{60}$ & 1930 & 1950 & $1960^{60}$ & 1930 & 1950 & $1960^{60}$ \\
Lutry & 29,3 & 26 & 17,3 & 27 & 27,6 & 31,5 & 43,6 & 46,4 & 51 \\
Savigny & 66 & 68,6 & 55 & 13,6 & 14,1 & 19,5 & 20,4 & 17,3 & 25,5
\end{tabular}

Au vu de cette répartition, il faut relever malgré sa nette diminution la part importante que garde le secteur primaire dans la commune de Savigny. La part du tertiaire a cependant nettement augmenté, cette dernière décade surtout 17,3 en 1950, 25,5 en 1960. Ni l'importance de la population, ni l'administration communale, ni le monde des affaires de la région ne justifient une part si importante du secteur tertiaire. Il faut donc en déduire qu'une partie de la population indigène a passé du secteur primaire au secteur tertiaire grâce à la proximité de l'agglomération lausannoise et qu'une population minime certes, mais dont il faut tenir compte, travaillant à Lausanne est venue se substituer à celle qui désertait la campagne. On ne peut pas encore parler du phénomène de banlieue comme à Lutry, mais il existe cependant un processus de transformation en cours. Le secteur secondaire constitué par un artisanat local a varié conformément au développement du tertiaire.

Lutry présente, elle, une répartition très différente: disparition progressive et accélerée, durant la dernière décade, du secteur primaire due essentiellement à l'extension de l'agglomération lausannoise qui maintenant élimine la vigne dans toute la partie ouest de la commune; montée du secteur tertiaire due à l'établissement d'une population importante cette fois et travaillant à Lausanne et aussi dans une moindre mesure à l'établissement d'un appareil administratif important causé par le développement local. Le secteur secondaire a tendance à s'accroître mais dans des proportions moindres et si on analyse la nature des entreprises on s'aperçoit qu'elle est artisanale. En effet, la seule entreprise de Lutry à occuper plus de cinquante personnes est un commerce de vin. La seconde en importance est une entreprise de construction qui compte 42 ouvriers. Deux autres entreprises occupent entre 20 et 30 personnes. Ces quatre maisons fournissent du travail aux $56 \%$ de la main-d'œuvre industrielle travaillant dans la commune, le $44 \%$ restant se répartissant sur 26 entreprises à raison de 5 ouvriers par entreprise en moyenne. L'industrie ne s'implantera pas à Lutry, tel est le vœux des autorités.

60 D'après le fichier du contrôle des habitants. 


\section{Population et implantation actuelle}

Il nous reste maintenant à analyser les incidences dues au dépeuplement à Savigny ou à l'essor démographique à Lutry sur les aménagements communaux. L'exode rural à Savigny a provoqué une diminution du nombre des exploitations et une augmentation de leur superficie.

\section{Savigny 1939: 167 exploitations 1955: 135 exploitations}

Savigny: Nombre d'exploitations agricoles (grandeur, surface en \% de chacune des catégories par rapport au total).

1939

1955

$\begin{array}{ccccccccc}\text { Expl. } & \text { ha } 0-1 & \% & \text { ha } 1,01-5 & \% & \text { ha } 5,01-10 & \% & \text { ha } 10,1 & \% \\ 167 & 17 & 10,1 & 44 & 26,4 & 68 & 40,7 & 38 & 22,8 \\ 135 & 8 & 5,9 & 27 & 20 & 49 & 36,4 & 51 & 37,7\end{array}$

La conséquence inévitable fut une mécanisation rapide, d'autant plus rapide que la main-d'œuvre même étrangère est actuellement introuvable dans le secteur agricole aux alentours de Lausanne. Les innombrables chantiers travaillant à l'exposition et à l'autoroute en sont les principales causes.

\begin{tabular}{clr} 
Savigny: 1939 & tracteurs & 5 \\
& machines à moteur & 18 \\
1955 & tracteurs & 35 \\
& machines à moteur & 29 \\
\multirow{2}{*}{1961} & tracteurs (2 essieux) & 61 \\
& tracteurs (1 essieu) & 15
\end{tabular}

Cette mécanisation n'allait pas sans entraîner le renforcement et la réfection d'un ancien réseau de chemins et de routes fort ramifié du fait de l'habitat dispersé. Entreprise coûteuse si l'on en juge par les sommes investies annuellement au poste des routes: (fr.)

\begin{tabular}{|c|c|}
\hline Savigny & $\begin{array}{c}1952 \\
22000\end{array}$ \\
\hline
\end{tabular}

Ce réseau a d'ailleurs tendance à se ramifier de plus en plus. En effet, malgré la diminution de la population, les charges communales n'ont fait qu'augmenter; situation passagère due à un sous-équipement devenu intolérable ou conséquence de l'établissement de résidences secondaires? Les deux probablement. N'oublions pas que les châlets de week-end causent passablement de frais à la communauté après que les particuliers ont fait leurs affaires; et les quarante résidences secondaires qui se sont construites depuis peu d'années sur le territoire communal ont nécessité une certaine mise de fonds. L'habitat dispersé joue également un rôle pour l'alimentation en eau; jusqu'à présent c'était plus une affaire de source privée que de réseau communal. Si l'on regarde les ressources de la commune on constate que le réseau débite journellement 57600 litres alors que les fontaines en fournissent 1872000 litres.

Si l'on en croit le rapport de la Suisse de $1951^{61}$ il semble que les $90 \%$ de la population suisse sont raccordés à un réseau de distribution d'eau. Savigny, à cet égard, douze ans plus tard, est encore légèrement au-dessous de ce pourcentage avec $86 \%$.

Dans le domaine hospitalier et médical, Savigny est tributaire de l'extérieur, aucun médecin, ni médecin-dentiste ne pratique sur la commune.

61 Union Internationale des Villes. Congrès de Brighton juin 1951. L'alimentation en eau et les réseaux d'égouts. La Haye 1951. 
Quant enfin au point de vue culturel et sportif la commune de Savigny doit lutter contre le lourd handicap qu'est l'habitat dispersé. La participation collective des habitants à telle ou telle manifestation est beaucoup plus difficile qu'ailleurs et ceci d'autant plus que pendant l'hiver, saison propice aux soirées pour les paysans, les déplacements sont rendus difficiles par la neige. Il n'y a guère que l'église qui sur le plan religieux réunisse régulièrement la communauté. Les écoles sont réparties sur le territoire communal. Celle de l'agglomération principale est récente; on a profité d'aménager par la même occasion une grande salle pour conférences ot spectacles. Même difficulté à réunir la population sur le plan sportif; il n'existe ni stade, ni club de football ou de basketball. Il y aurait peut-être dans ce domaine quelques lacunes à combler. La création généralisée de piscines dans les différentes communes du canton s'avère être une réussite. La proximité de l'agglomération lausannoise devrait assurer à un établissement semblable sur la commune de Savigny une clientèle détournée de plus en plus du lac par la pollution des eaux du Léman et par la promiscuité souvent insupportable qui règne sur les rares plages publiques de Lausanne et des environs. Peut-être donnerait-on le départ ainsi à une petite vie touristique. La transformation de la piscine en une patinoire durant l'hiver semble climatiquement parlant tout-à-fait possible.

Disons pour terminer que la commune de Savigny bien qu'ayant diminué de population ces dernières années, risque probablement de subir lentement l'influence de l'agglomération lausannoise. Il lui faudra, il lui faudrait déjà pour éviter des erreurs de goût de plus en plus fréquentes et pour freiner une prolifération anarchique de nouvelles maisons et de résidences secondaires, instaurer un règlement sur la police de construction et faire approuver un plan de zone ayant force légale. Ces mesures auraient pour conséquences de protéger la beauté du paysage et d'offrir à l'agriculture un vaste champs d'action pour se transformer en cas de nécessité économique (crise ou intégration).

Si le problème de l'implantation ne se pose que de nos jours pour la commune de Savigny, il n'en va pas de même pour Lutry. Dès 1920 commence une prolifération nouvelle de l'habitat le long du lac dans la région ouest de la commune. De nature dispersée, il va peu à peu gravir la pente et occuper tout le versant, chassant progressivement la vigne dans ce secteur. Après la dernière guerre mondiale le phénomène prend une ampleur très vaste. Bien desservie au point de vue communication, Lutry devenait rapidement après Pully dans la direction est, la commune la plus recherchée par des gens travaillant à Lausanne et voulant habiter hors de ville. Tranquillité, situation privilégiée, modicité relative des loyers en comparaison de la capitale attiraient de plus en plus de monde.

Construction durant la période 1953-1962

\begin{tabular}{|c|c|c|c|c|c|c|c|c|c|c|c|c|}
\hline & \multicolumn{3}{|c|}{1953} & \multicolumn{3}{|c|}{1954} & \multicolumn{3}{|c|}{1955} & \multicolumn{3}{|c|}{1956} \\
\hline & I & II & III & I & II & III & I & II & III & I & II & III \\
\hline \multirow{3}{*}{$\begin{array}{l}\text { Savigny } \\
\text { Lutry }\end{array}$} & - & - & - & 6 & 6 & 19 & 一 & - & - & - & - & - \\
\hline & 15 & 10 & 48 & 19 & 9 & 60 & 15 & 15 & 75 & 26 & 17 & 100 \\
\hline & & 1957 & & & 195 & & & 1959 & & & 1960 & \\
\hline \multirow{4}{*}{$\begin{array}{l}\text { Savigny } \\
\text { Lutry }\end{array}$} & & & 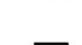 & 5 & 5 & ? & 3 & & 8 & 5 & & 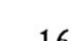 \\
\hline & 43 & 16 & 174 & 11 & 10 & 46 & 21 & 21 & 94 & 15 & 15 & 66 \\
\hline & & 1961 & & tot: & 195 & & & & & & & \\
\hline & I & II & III & I & II & III & & & & & & \\
\hline Savigny & 4 & 4 & 14 & 23 & 21 & 77 & & & & & & \\
\hline Lutry & 33 & 17 & 137 & 188 & 130 & 80062 & & & & & & \\
\hline
\end{tabular}

62 I nombre de logements, II dont maisons familiales, III nombre des pièces. 
Devant cette situation qui portait déjà atteinte à un paysage d'une beauté certaine et qui risquait de supprimer le caractère même de la cité de Lutry et celui des hameaux vignerons, la commune a établi «un règlement sur le plan d'extension et la police de construction» dès 1959. Un plan divise le territoire communal en six zones:
1) zone de l'ord re contigu
2) zone de l'ordre non-contigu
3) zone des villas
4) zone viticole et zone rurale
5) zone de la vieille ville
6) zone de verdure

Si les zones 1 et 2 font l'objet de mesures d'ordre général, certains articles concernant les zones 3,4 et 5 sont stricts et prouvent le réel souci qu'ont les autorités communales quant au développement de leur cité.

Des établissements artisanaux ou commerciaux peuvent exceptionnellement y être autorisés pour autant qu'ils ne portent pas préjudice à l'habitation ou qu'ils ne compromettent pas le caractère du quartier. Le respect du cadre et du paysage en général est souligné dans les articles concernant la zone viticole et rurale tandis qu'on insiste sur les proportions des bâtiments qui doivent être conformes aux anciennes maisons dans les articles consacrés à la zone de la vieille ville. Des règles spéciales empêchant pratiquement l'établissement d'industries, la construction des châlets au bord du lac ainsi que des pavillons et maisons de week-end complètent ce règlement de police. Trois articles consacrés à l'esthétique des constructions donnent aux autorités la possibilité d'accepter ou de refuser certains projets extravagants et d'interdire certaines teintes de façades.

Après avoir établi la répartition des zones, le service des travaux de la ville de Lutry s'est efforcé d'établir un plan de quartiers pour les régions situées entre la ligne du Simplon et la route cantonale Lausanne-Brigue, qui sont les plus convoitées pour la construction. Devant l'augmentation très rapide de la population qui risquait en s'établissant selon la formule de la cité-jardin d'occuper dangereusement le territoire de la commune, les autorités, à une faible majorité il est vrai, avaient opté pour la création de grands complexes qui auraient eu comme gros avantages d'absorber une population importante (2000 personnes). Cette création aurait semble-t-il évité, indirectement le fractionnement du terrain tout en tendant à une occupation, à une répartition et à une exploitation rationnelles du sol.

La population autochtone choquée par les dimensions d'un des bâtiments qui s'étendait sur quelques centaines de mètres parallèlement au lac, effrayée peut-être aussi par l'apport subit d'un effectif qui aurait doublé la population de la cité s'est opposée par voie de referendum et a réussi à faire échouer le projet.

Quelle que soit sa forme, le développement de Lutry semble irrémédiable à moins d'événements difficilement prévisibles. Il s'agit de voir sommairement dans quelle mesure la commune se prépare à cette évolution au point de vue équipement.

La position de Lutry sur un grand axe de circulation implique aujourd'hui d'importants aménagements. Jusqu'à la fin du XIXe siècle le trafic était surtout interne et s'établissait selon un axe Nord-Sud, mais à partir de ce moment là, le trafic externe orienté Est-Ouest prit petit-à-petit le pas. La motorisation permet une augmentation des échanges, une généralisation progressive des déplacements et des déplacements quotidiens de nature professionnelle avant tout (mouvements pendulaires).

De nos jours, la motorisation très poussée de la population résidente (5,5 habitants par voiture), la vogue touristique de l'ensemble du bassin lémanique et l'intensification du trafic international très prochaine due à l'ouverture en 1964 du Tunnel du Grand Saint-Bernard obligent la commune de Lutry à réaménager son réseau routier.

Après s'être consacrée ces dernières années au revêtement des chemins, pratiquant des investissements énormes mais rentables à la longue puisqu'ils suppriment la remise en état annuelle des chemins ravinés, la commune entreprend maintenant l'élargissement de la route nationale actuelle en bordure du lac. De plus, elle va prochainement voir s'ouvrir sur son territoire, à la hauteur de la route des Monts, l'autoroute Lausanne-Brigue. 
La présence de la station de pompage de la ville de Lausanne sur la commune de Lutry ainsi que le passage sur les Hauts de la conduite venant de l'Etivaz et desservant également les habitants de Lausanne évitent bien des tracas. Un problème beaucoup plus inquiétant est celui des eaux usées. La densité de la population qui augmente en même temps que la pollution du lac interdit toute insouciance. Les terrains pour la station d'épuration sont achetés, les plans approuvés, la construction va commencer sous peu.

L'équipement scolaire après avoir été durant des années déficient est depuis environ quatre ans plus que satisfaisant. Lors de la construction des nouveaux bâtiments du collège on a profité d'aménager une grande place de sport, chose encore assez rare en Romandie pour qu'on le relève. Sur le plan d'extension de la ville de Lutry, nous avons pu voir que certains terrains étaient déjà prévus pour de futurs bâtiments scolaires que nécessitera l'essor plus ou moins rapide de Lutry.

Une analyse détaillée des services de la ville de Lutry nous entrainerait trop loin. Il faut cependant remarquer qu'à cinq kilomètres de Lausanne, la population trouve sur place médecins, médecins-dentistes, pharmacien, banque, commerce de tous genres, bien montés, énumération qui montre la vitalité de la cité et qui lui confère encore une autre valeur que celle d'une cité dortoir.

Le dernier chapitre, sommaire, de notre étude, nous amène à comprendre que notre époque est une nouvelle période de complète transformation pour la région choisie. Dans le jeu subtil des multiples facteurs entre eux, il en est un actuellement qui bouscule tous les autres; c'est la poussée démographique. Le sol qui était encore au début du siècle un champ à cultiver est devenu un vaste terrain à bâtir. Lentement, la conscience du vigneron et du paysan s'est transformée. De conscience d'exploitant terrien elle devient conscience de propriétaire foncier.

Nous remarquons cette évolution sans la juger. Nous relèverons cependant qu'elle porte en elle deux menaces; la première qui consiste à laisser disparaître le secteur primaire, essentiel non seulement à une économie de guerre mais bien plus à une société équilibrée, la seconde qui est la détérioration d'un paysage humanisé. Lavaux représente un effort de plusieurs siècles et il a fait naître dans le domaine culturel des œuvres qui sont parmi les plus significatives de notre pays; le voir peu à peu disparaître, c'est également se demander quel sera son nouvel aspect. Subir sa transformation ou l'orienter? Seule une connnaissance approfondie de l'évolution permettra que la prise de conscience générale soit suivie de décisions conformes à l'intérêt de la région et de sa population, constituant un nouveau plan d'aménagement du territoire.

\section{ZUR ORTSPLANUNG ZWEIER WAADTLÄNDER GEMEINDEN : LUTRY UND SAVIGNY}

Das Studium der wichtigsten Faktoren, die der Ausarbeitung einer Landesplanung - wenn auch nur für ein kleines Gebiet - vorangegangen sind, offenbart häufig unerwartete Vorgänge und trägt manchmal zum tieferen Verständnis einer Entwicklung bei, die man sich heute immer mehr zu überblicken bemüht.

Um zu verstehen, daß Lutry und Savigny, zwei benachbarte Gemeinden, die früher ein politisches und wirtschaftliches Ganzes bildeten, heute keinerlei Verbindung mehr haben, muß man auf die naturgegebenen Bedingungen zurückkommen und ihre bisherige Nutzbarmachung durch den Menschen studieren. Zuerst muß auf die Relief- und Klimaunterschiede der beiden Gebiete hingewiesen werden. Dann ist die zunehmende wirtschaftliche Wichtigkeit des Weinbaues seit dem 1o. Jh. zu unterstreichen, da dieser für die Bevölkerung eine vollständige Veränderung der Lebensweise mit sich brachte. Die Nutzung des Hinterlandes der Gemeinde Lutry sowie dessen Kolonisierung sind die direkten Folgen der raschen Entwicklung des Weinbaues. Bezüglich der Teilung der Gemeinde muß man sich ebenfalls über die tieferen Gründe dieser Entscheidung befragen. Und schließlich muß man die heutige Lage dieser zwei Gebiete im Verhältnis zu den großen Verkehrsadern betrachten, um die sehr unterschiedliche Situation der beiden Gemeinden gegenüber der Ausdehnung der Lausanner Siedlung erklären zu können.

Ein solches Vorgehen hat zwei Vorteile. - Einerseits ermöglicht es die Erklärung der «réalités territoriales», die für die beiden Gemeinden sehr verschieden sind, andererseits gibt es die geschichtlichen Abschnitte wieder, die diese Gemeinden durch wirtschaftliche, juristische und soziale Maßnahmen beeinflußt haben.

Dieses Vorgehen kann vielleicht auch bei anderen Weinbaugemeinden angewendet werden. 